\title{
Measure Theory in Noncommutative Spaces ${ }^{\star}$
}

Steven $L O R D^{\dagger}$ and Fedor SUKOCHEV ${ }^{\ddagger}$

$\dagger$ School of Mathematical Sciences, University of Adelaide, Adelaide, 5005, Australia

E-mail: steven.lord@adelaide.edu.au

$¥$ School of Mathematics and Statistics, University of New South Wales, Sydney, 2052, Australia E-mail: f.sukochev@unsw.edu.au

Received March 25, 2010, in final form August 04, 2010; Published online September 16, 2010 doi:10.3842/SIGMA.2010.072

\begin{abstract}
The integral in noncommutative geometry (NCG) involves a non-standard trace called a Dixmier trace. The geometric origins of this integral are well known. From a measure-theoretic view, however, the formulation contains several difficulties. We review results concerning the technical features of the integral in NCG and some outstanding problems in this area. The review is aimed for the general user of NCG.
\end{abstract}

Key words: Dixmier trace; singular trace; noncommutative integration; noncommutative geometry; Lebesgue integral; noncommutative residue

2010 Mathematics Subject Classification: 46L51; 47B10; 58B34

\section{Contents}

1 Introduction $\quad 2$

2 Dixmier traces $\quad \mathbf{5}$

2.1 Singular symmetric functionals $\ldots \ldots \ldots \ldots \ldots \ldots$

3 Measurability 11

3.1 Decomposition of measurable operators . . . . . . . . . . . . . . . . 13

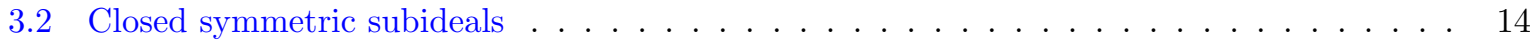

4 Origin of the noncommutative integral $\quad 15$

4.1 Normal extension . . . . . . . . . . . . . . . . . . . . . . . . . . . 17

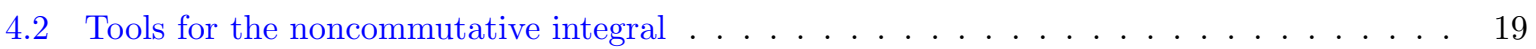

5 Zeta functions and heat kernels $\quad \mathbf{2 0}$

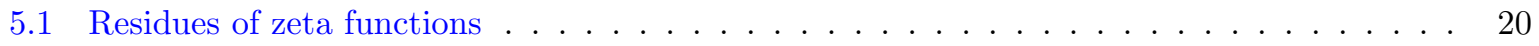

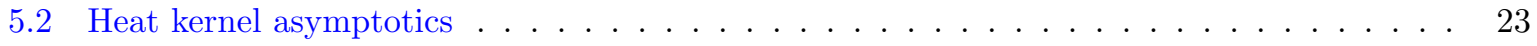

6 Characterising the noncommutative integral $\quad \mathbf{2 4}$

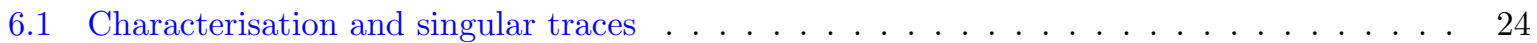

6.2 A dominated convergence theorem for the noncommutative integral . . . . . . . . . . . 27

7 Summary $\quad \mathbf{2 7}$

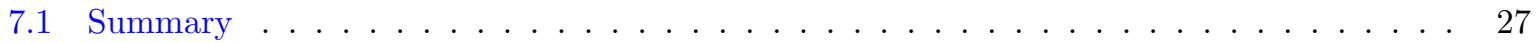

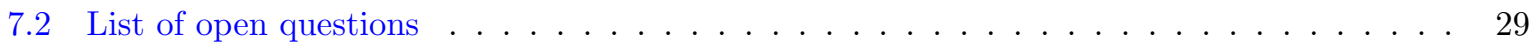

$\begin{array}{ll}\text { A Identifications in (2.37) } & 29\end{array}$

$\begin{array}{ll}\text { References } & 34\end{array}$

${ }^{\star}$ This paper is a contribution to the Special Issue "Noncommutative Spaces and Fields". The full collection is available at http://www.emis.de/journals/SIGMA/noncommutative.html 


\section{Introduction}

The basic object of noncommutative geometry (NCG) is a spectral triple $(\mathcal{A}, H, D)$ [1], originally called a $\mathrm{K}$-cycle $[2$, p. 546]. Here $\mathcal{A}$ is a countably generated unital non-degenerate $*$-algebra of bounded linear operators on a separable complex Hilbert space $H$, and $D: \operatorname{Dom} D \rightarrow H$ is a selfadjoint linear operator with the properties $\|[D, a]\|<\infty$ for all $a \in \mathcal{A}$ and $\langle D\rangle^{-1}:=$ $\left(1+D^{2}\right)^{-\frac{1}{2}}$ is a compact operator.

In Connes' quantised calculus the compact operator $\langle D\rangle^{-1}$ is the analogue of an infinitesimal length element $d s$ [2, p. 545], [3, p. 157], and the integral of $a \in \mathcal{A}$ is represented by the operator-theoretic expression [2, p. 545], [3, p. 158],

$$
\operatorname{Tr}_{\omega}\left(a\langle D\rangle^{-n}\right), \quad a \in \mathcal{A},
$$

where $n$ (assumed to exist) is the smallest value such that $\langle D\rangle^{-p}$ is trace class for $p>n$, and the positive trace $\operatorname{Tr}_{\omega}$ (nominally a 'Dixmier trace') measures the log divergence of the trace of $a\langle D\rangle^{-n}$. The link between integration in the traditional sense and sums of eigenvalues of the product $a\langle D\rangle^{-n}$ is not obvious.

Segal, in [4], formalised an operator-theoretic view of integration theory. The subsequently developed theory ${ }^{1}[5,6]$, through to $[7,8,9]$, features the notion of a faithful normal trace $\tau$ on a $*$-algebra $\mathcal{N}$ of bounded linear operators on $H$ that is closed in the weak operator topology (a von Neumann algebra). The trace $\tau$ is used to build an $L^{p}$-theory with $L^{\infty}(\mathcal{N}, \tau)=\mathcal{N}$ and $L^{p}(\mathcal{N}, \tau)$ being the completion of the set $\left\{x \in \mathcal{N} \mid \tau\left(|x|^{p}\right)<\infty\right\}$. The trace (and the integration theory) is called finite if $\tau\left(1_{\mathcal{N}}\right)<\infty$ where $1_{\mathcal{N}}$ is the identity of $\mathcal{N}$. The integration theory associated to the pair $(\mathcal{N}, \tau)$ has analogous machinery to measure theory, including Radon-Nikodym theorems, dominated and monotone convergence theorems ${ }^{2}$, etc. Without the condition of normality this machinery fails.

A commutative example of the theory (and the prototype) is a regular Borel measure space $(X, \mu)$. For the von Neumann algebra of complex functions $f \in L^{\infty}(X, \mu)$ acting on the separable Hilbert space $L^{2}(X, \mu)$ by (almost everywhere) pointwise multiplication, the integral

$$
\tau_{\mu}(f)=\int_{X} f d \mu, \quad f \in L^{\infty}(X, \mu)
$$

is a faithful normal trace. The noncommutative $L^{p}$-spaces are the usual $L^{p}$-spaces, i.e.

$$
L^{p}\left(L^{\infty}(X, \mu), \tau_{\mu}\right) \equiv L^{p}(X, \mu) .
$$

A noncommutative example is the von Neumann algebra $\mathcal{L}(H)$ of bounded linear operators on a separable Hilbert space $H$. The canonical trace

$$
\operatorname{Tr}(T)=\sum_{m=1}^{\infty}\left\langle h_{m}, T h_{m}\right\rangle, \quad T \in \mathcal{L}(H),
$$

where $\left\{h_{m}\right\}_{m=1}^{\infty}$ is an orthonormal basis of $H$, is a faithful normal trace. The resulting $L^{p}$-spaces

$$
\mathcal{L}^{p}(H):=\left\{T \in \mathcal{L}(H) \mid \operatorname{Tr}\left(|T|^{p}\right)<\infty\right\}
$$

\footnotetext{
${ }^{1}$ The explanation here is for semifinite von Neumann algebras with separable pre-dual.

${ }^{2}$ The condition of normality of a linear functional preempts the monotone convergence theorem. If $\mathcal{N}$ is a closed $*$-subalgebra of $\mathcal{L}(H)$ in the weak operator topology then a linear functional $\rho \in \mathcal{N}^{*}$ is normal if $\rho(S)=\sup _{\alpha} \rho\left(S_{\alpha}\right)$ for all increasing nets of positive operators $\left\{S_{\alpha}\right\} \subset \mathcal{N}$ in the strong operator topology with l.u.b. $S$. This condition is equivalent to $\rho\left(\sum_{i} P_{i}\right)=\sum_{i} \rho\left(P_{i}\right)$ for all sets $\left\{P_{i}\right\}$ of pairwise orthogonal projections from $\mathcal{N}$ [10, p. 67], i.e. analogous to additivity of a measure on a $\sigma$-algebra of sets.
} 
are the Schatten-von Neumann ideals of compact operators [11] ([12] for finite dimensional $H$ ). A consequence of the Radon-Nikodym theorem in this context is that for any normal linear functional $\phi$ on a von Neumann algebra $\mathcal{N} \subset \mathcal{L}(H)$ there is a trace class 'density' $T \in \mathcal{L}^{1}(H)$ such that, [10, p. 55],

$$
\phi(a)=\operatorname{Tr}(a T), \quad \forall a \in \mathcal{N} .
$$

Combining the examples, for every regular Borel measure $\mu$ on a space $X$ there is a positive trace class operator $T_{\mu}$ on the Hilbert space $L^{2}(X, \mu)$ such that

$$
\int_{X} f d \mu=\operatorname{Tr}\left(f T_{\mu}\right), \quad f \in L^{\infty}(X, \mu) .
$$

This formula exhibits the standard association between integration and the eigenvalues of the product $f T_{\mu}$, a compact linear operator.

What are the differences between (1.1), the integral in NCG, and (1.3), the integral according to standard noncommutative integration theory? Let us mention basic facts about (1.1).

Firstly, since $\langle D\rangle^{-n}$ belongs to the ideal of compact linear operators

$$
\mathcal{M}_{1, \infty}(H):=\left\{T \in \mathcal{L}^{\infty}(H) \mid \sup _{n \in \mathbb{N}} \frac{1}{\log (1+n)} \sum_{k=1}^{n} \mu_{k}(T)<\infty\right\}
$$

(where $\mathcal{L}^{\infty}(H)$ denotes the compact linear operators and $\mu_{n}(T), n \in \mathbb{N}$, are the eigenvalues of $|T|=\left(T^{*} T\right)^{1 / 2}$ arranged in decreasing order), and the positive traces $\operatorname{Tr}_{\omega}$ are linear functionals on $\mathcal{M}_{1, \infty}$, the formula

$$
\operatorname{Tr}_{\omega}\left(S\langle D\rangle^{-n}\right), \quad S \in \mathcal{L}(H)
$$

defines a linear functional on $\mathcal{L}(H)$. In particular, by restriction (1.6) is a linear functional on the *-algebra $\mathcal{A}$, on the closure $A$ of $\mathcal{A}$ in the uniform operator topology (a separable $C^{*}$ subalgebra of $\mathcal{L}(H)$ since $\mathcal{A}$ is countably generated), and on the closure $\mathcal{A}^{\prime \prime}$ of $\mathcal{A}$ in the weak (or strong) operator topology (the bicommutant, a von Neumann subalgebra of $\mathcal{L}(H)[13, \S 2.4]$ ).

Secondly, if $0<S \in \mathcal{L}(H)$,

$$
\operatorname{Tr}_{\omega}\left(S\langle D\rangle^{-n}\right)=\operatorname{Tr}_{\omega}\left(\sqrt{S}\langle D\rangle^{-n} \sqrt{S}\right) \geq 0 .
$$

Hence (1.6) defines a positive linear functional on $\mathcal{A}, A$ or $\mathcal{A}^{\prime \prime}$.

Thirdly,

$$
\left|\operatorname{Tr}_{\omega}\left(S\langle D\rangle^{-n}\right)\right| \leq C\|S\|, \quad \forall S \in \mathcal{L}(H)
$$

for a constant $C>0$. Hence the same formula (1.6) is the unique uniformly continuous extension to $A$ and $\mathcal{A}^{\prime \prime}$ of the positive linear functional (1.1) on $\mathcal{A}$. Denote this linear functional by $0<\Phi_{\omega} \in\left(\mathcal{A}^{\prime \prime}\right)^{*}$.

Fourthly, $\Phi_{\omega}$ is a trace on $\mathcal{A}^{\prime \prime}[14$, Theorem 1.3], [2, p. 313], [15, p. 280], [16, p. 47].

The fundamental difference between the functionals (1.3) and (1.6) is that $\operatorname{Tr}_{\omega}\left(S\langle D\rangle^{-n}\right)=0$ for all positive finite rank operators $S$ (see Section 2 below). In comparison $\operatorname{Tr}(S T)>0$ for a trace class operator $0<T \in \mathcal{L}^{1}(H)$ with trivial kernel and every finite rank operator $S>0$. This property of vanishing on the finite rank operators, called singularity, is essential for the fundamental theorem of integration theory in NCG (Connes' trace theorem [17], and Theorem 4.1 in this text). The property of singularity, however, implies that the functional (1.6) is either trivial or else is a non-normal functional on $\mathcal{L}(H)$. To see this, let $S_{n}$ be a sequence of positive 
finite rank operators converging upward strongly to the identity 1 of $\mathcal{L}(H)$ and suppose (1.6) is a normal linear functional on $\mathcal{L}(H)$, then

$$
0=\sup _{n} \operatorname{Tr}_{\omega}\left(S_{n}\langle D\rangle^{-n}\right)=\operatorname{Tr}_{\omega}\left(\langle D\rangle^{-n}\right) .
$$

Hence either $\operatorname{Tr}_{\omega}\left(\langle D\rangle^{-n}\right)=0$ or else (1.6) is non-normal.

The property of singularity does not exclude the trace $0<\Phi_{\omega} \in\left(\mathcal{A}^{\prime \prime}\right)^{*}$ (the restriction of (1.6) to the von Neumann algebra $\mathcal{A}^{\prime \prime} \subset \mathcal{L}(H)$ ) from being a normal linear functional on $\mathcal{A}^{\prime \prime}$. There are spectral triples where $\Phi_{\omega}$ is not a normal linear functional on $\mathcal{A}^{\prime \prime}$ (evidently when $\mathcal{A}^{\prime \prime}$ contains a finite rank operator), and where $\Phi_{\omega}$ is a normal linear functional on $\mathcal{A}^{\prime \prime}$ (see Theorem 4.3).

Currently we lack, in general, a characterisation of the relationship between $D$ and $\mathcal{A}$ that implies $\Phi_{\omega} \in\left(\mathcal{A}^{\prime \prime}\right)^{*}$ is a normal linear functional. All we know of is a sufficient condition, due to the authors, see Theorem 6.2 in Section 6.2. It has been suggested that $\mathcal{A}^{\prime \prime}$ containing no finite rank operators is a necessary and sufficient condition for normality of $\Phi_{\omega}$. This conjecture is open. Non-normality of the linear functional on $\mathcal{L}(H)$ defined by (1.6) can have advantages, as indicated by Connes [2, p. 326]. However, normality of the trace $0<\Phi_{\omega} \in\left(\mathcal{A}^{\prime \prime}\right)^{*}$ is coincident with our ability to apply the standard tools of integration theory to the formula (1.1).

In general, the value of the trace $0<\Phi_{\omega} \in\left(\mathcal{A}^{\prime \prime}\right)^{*}$ on the projections of $\mathcal{A}^{\prime \prime}$,

$$
\Phi_{\omega}(E), \quad E^{*}=E=E^{2} \in \mathcal{A}^{\prime \prime},
$$

is only analogous to an element of the ba space of finite and finitely additive functionals on a Borel $\sigma$-algebra [18, IV.2]. The integral (1.1) is constructed by limits of finite linear combinations of the values in (1.7) if and only if the trace $\Phi_{\omega}$ is normal.

In this review, we discuss the known ways to measure the 'log divergence of the trace', including residue formulas, heat kernels and Dixmier traces. Each of these ways may result in different functionals in (1.1). It is desirable to know when all these ways agree, and that (1.1) defines a unique linear functional on $\mathcal{A}$. There are a variety of difficulties behind this problem. For example, if $\mathcal{V}$ denotes a set of linear functionals on $\mathcal{M}_{1, \infty}$ 'measuring the log divergence of the trace', the set

$$
\mathcal{K}_{\mathcal{V}}:=\left\{T \in \mathcal{M}_{1, \infty} \mid \mathrm{f}(T)=\text { const } \forall \mathrm{f} \in \mathcal{V}\right\}
$$

is not an ideal [19, Remark 3.6]. Therefore, $\langle D\rangle^{-n} \in \mathcal{K}_{\mathcal{V}}$ will not imply $a\langle D\rangle^{-n} \in \mathcal{K}_{\mathcal{V}}$ for all $a \in \mathcal{A}$ in general. Specifying $\langle D\rangle^{-1}$ alone is not sufficient for a unique value from the formula (1.1). Additionally, if one picks smaller sets, $\mathcal{V}$, or larger sets, $\mathcal{V}^{\prime}$, of linear functionals, it may be that $\mathcal{K}_{\mathcal{V}} \neq \mathcal{K}_{\mathcal{V}^{\prime}}$ (discussed in Section 3). Allied to this problem is the fact that, if $a \in \mathcal{A}$ is a selfadjoint operator and $a\langle D\rangle^{-n} \in \mathcal{K}_{\mathcal{V}}$ it is not necessarily true that the decompositions $a_{+}\langle D\rangle^{-n}, a_{-}\langle D\rangle^{-n} \in \mathcal{K}_{\mathcal{V}}$, where $a_{+}$and $a_{-}$are the positive and negative parts of $a$.

Currently, in general, there is no characterisation, given $\langle D\rangle^{-n}$, what relationship with a $*$-algebra $\mathcal{A}$ is necessary so that $\Phi_{\omega}(a)=\operatorname{Tr}_{\omega}\left(a\langle D\rangle^{-n}\right)$ specifies a unique trace (meaning the value of $\Phi_{\omega}(a)$ is independent of the linear functional $\left.\operatorname{Tr}_{\omega} \in \mathcal{V}\right)$. This is an open problem. Checking uniqueness is a case by case basis which requires identifying $\operatorname{Tr}_{\omega}\left(a\langle D\rangle^{-n}\right)$ with a known functional, e.g. the Lebesgue integral for a compact Riemannian manifold [17, 16], or the Hausdorff measure of Fuchsian circles and self-similar fractals [2, IV.3], [20], or estimating the singular values of the product $a\langle D\rangle^{-n}$.

In the review we shall provide some sufficient conditions for normality of the trace $0<$ $\Phi_{\omega} \in\left(\mathcal{A}^{\prime \prime}\right)^{*}$. We review the demonstration of normality for the case of a compact Riemannian manifold (in Section 4) and the noncommutative torus (in Section 6).

Finally, we discuss the role of other positive singular traces on $\mathcal{M}_{1, \infty}$, besides Dixmier traces, in the formula (1.1). We show that, even in Connes' trace theorem [17, Theorem 1], there exist 
other positive singular traces $\rho$ on the compact operators, which are not Dixmier traces, such that $\rho(P)=\operatorname{Res}(P)$ (up to a positive constant) for a classical pseudo-differential $P$ of order $-n$ on a $n$-dimensional compact Riemannian manifold, where Res is the Wodzicki residue.

\section{Notation}

Throughout $H$ denotes a separable complex Hilbert space, $\mathcal{L}(H)$ (resp. $\mathcal{L}^{\infty} \equiv \mathcal{L}^{\infty}(H)$, $\mathcal{L}^{p} \equiv \mathcal{L}^{p}(H), p \in[1, \infty)$ ) denotes the linear bounded (resp. compact, Schatten-von Neumann (see (1.2))) operators on $H$. Throughout the text $\mathcal{M}_{1, \infty} \equiv \mathcal{M}_{1, \infty}(H)$ denotes the ideal of compact operators defined in (1.5) or (2.2) below. Denote by $\ell^{\infty}$ (resp. $c, c_{0}, \ell^{p}$ ) the bounded (resp. convergent, convergent to zero, $p$-summable) sequences. Similarly, for a regular Borel measure space $(X, \mu), L^{\infty}(X, \mu)$ (resp. $\left.L^{p}(X, \mu), C_{b}(X), C_{0}(X)\right)$ denotes the essentially bounded (resp. $p$-integrable, continuous and bounded, continuous and vanishing at infinity) (equivalence classes of) functions on $X$. When $X$ is a Riemannian manifold and $d x$ is the volume form on $X$, we write $L^{p}(X) \equiv L^{p}(X, d x)$.

\section{Dixmier traces}

For a separable complex Hilbert space $H$, denote by $\mu_{n}(T), n \in \mathbb{N}$, the singular values of a compact operator $T$ (the singular values are the eigenvalues of the operator $|T|=\left(T^{*} T\right)^{1 / 2}$ arranged with multiplicity in decreasing order $[11, \S 1])$. Define the logarithmic average

$$
\alpha\left(\left\{\mu_{n}(T)\right\}_{n=1}^{\infty}\right):=\left\{\frac{1}{\log (1+k)} \sum_{n=1}^{k} \mu_{n}(T)\right\}_{k=1}^{\infty}, \quad T \in \mathcal{L}^{\infty} .
$$

Then

$$
\mathcal{M}_{1, \infty}:=\mathcal{M}_{1, \infty}(H)=\left\{T \mid\|T\|_{1, \infty}:=\sup _{k} \alpha\left(\left\{\mu_{n}(T)\right\}_{n=1}^{\infty}\right)_{k}<\infty\right\}
$$

defines a Banach ideal of compact operators whose sequence of partial sums of singular values is of order logarithm. We refer to the recent paper of Pietsch [21], discussing the origin of this object in mathematics. In [22], J. Dixmier constructed a non-normal semifinite trace (a Dixmier trace) on $\mathcal{L}(H)$ using the weight

$$
\operatorname{Tr}_{\omega}(T):=\omega\left(\left\{\frac{1}{\log (1+k)} \sum_{n=1}^{k} \mu_{n}(T)\right\}_{k=1}^{\infty}\right), \quad T>0,
$$

where $\omega$ is a state on $\ell^{\infty}$ associated to a translation and dilation invariant state on $\mathbb{R}$. The sequence (2.1), while bounded for $T \in \mathcal{M}_{1, \infty}$, is not generally convergent. There are operators $0<T \in \mathcal{M}_{1, \infty}$ for which the sequence $\alpha\left(\left\{\mu_{n}(T)\right\}_{n=1}^{\infty}\right) \in c$ (called Tauberian operators), and for the Tauberian operators we want a Dixmier trace to be equal to the limit $\lim _{k \rightarrow \infty} \alpha\left(\left\{\mu_{n}(T)\right\}_{n=1}^{\infty}\right)_{k}$. However, the linear span of the set of Tauberian operators does not form an ideal of $\mathcal{M}_{1, \infty}$. For the semifinite domain of a Dixmier trace to be an ideal of compact operators, the use of a state $\omega$ on $\ell^{\infty}$ such that $\omega\left(a_{k}\right)=\lim _{k} a_{k}$ when $a_{k} \in c$ (known as a generalised limit) cannot be avoided. Note that $\left|\omega\left(a_{k}\right)\right| \leq\left\|a_{k}\right\|_{\infty}$, hence

$$
\left|\operatorname{Tr}_{\omega}(T)\right| \leq\|T\|_{1, \infty}, \quad T \in \mathcal{M}_{1, \infty} .
$$

A Dixmier trace $\operatorname{Tr}_{\omega}$ is positive by construction and continuous by $(2.4)$, i.e. $0<\operatorname{Tr}_{\omega} \in \mathcal{M}_{1, \infty}^{*}$. That it is a trace follows from the identity $\mu_{n}\left(U^{*} T U\right)=\mu_{n}(T)$ for any unitary $U \in \mathcal{L}(H)$. That 
it is singular (vanishes on finite rank operators, in fact on any trace class operator) follows from $\alpha\left(\left\{\mu_{n}(S)\right\}_{n=1}^{\infty}\right) \in c_{0}$ for any finite rank operator $S$ (resp. $S \in \mathcal{L}^{1}$ ). The basic facts stated in the introduction now follow (for the fourth see the cited references). For the third notice that $\|S T\|_{1, \infty} \leq\|S\|\|T\|_{1, \infty}$ (hence $\left|\operatorname{Tr}_{\omega}(S T)\right| \leq\|S\|\|T\|_{1, \infty}$ ) for all $S \in \mathcal{L}(H), T \in \mathcal{M}_{1, \infty}$.

The non-trivial feature of $\operatorname{Tr}_{\omega}$ is linearity. Dixmier's specification that $\omega$ is constructed from a translation and dilation invariant state on $\mathbb{R}$ provides sufficient conditions for linearity. Later formulations of the Dixmier trace considered wider or narrower specifications suited to their context.

Unfortunately, the specifications are a mess. We will now introduce a wide range of sets of linear functionals, all of which play some part in known results concerning the Dixmier trace.

Let $S\left(\ell^{\infty}\right)$ denote the states on $\ell^{\infty}$, i.e. the positive linear functionals $\sigma$ such that $\sigma((1,1, \ldots))$ $=1$. Let $S([1, \infty))($ resp. $S([0, \infty)))$ denote the states of $L^{\infty}([1, \infty))\left(\right.$ resp. $\left.L^{\infty}([0, \infty))\right)$. A state on $\ell^{\infty}$ (resp. $\left.L^{\infty}([1, \infty)), L^{\infty}([0, \infty))\right)$ is singular if it vanishes on finite sequences (resp. functions with a.e. compact support). It is easy to show a state $\sigma$ on $\ell^{\infty}\left(\operatorname{resp} . L^{\infty}([1, \infty)), L^{\infty}([0, \infty))\right)$ is singular if and only if

$$
\begin{aligned}
& \liminf _{n \rightarrow \infty} a_{n} \leq \sigma\left(\left\{a_{n}\right\}_{n=1}^{\infty}\right) \leq \limsup _{n \rightarrow \infty} a_{n}, \quad a_{n}>0 \quad \forall n \in \mathbb{N} \\
& \text { (resp. ess- } \left.\liminf _{t \rightarrow \infty} f(t) \leq \sigma(f) \leq \text { ess- } \limsup _{t \rightarrow \infty} f(t), \quad f>0\right) .
\end{aligned}
$$

That is, a state is singular if and only if it is an extension to $\ell^{\infty}\left(\right.$ resp. $\left.L^{\infty}([1, \infty)), L^{\infty}([0, \infty))\right)$ of the ordinary limit of convergence sequences (resp. limit at infinity of convergent at infinity functions). The Hahn-Banach theorem guarantees that these linear extensions, called generalised limits, exist. Denote by $S_{\infty}\left(\ell^{\infty}\right)\left(\right.$ resp. $\left.S_{\infty}([1, \infty)), S_{\infty}([0, \infty))\right)$ the set of singular states (or generalised limits). Denote by $S C_{\infty}\left([1, \infty)\right.$ ) (resp. $\left.S C_{\infty}([0, \infty))\right)$ the same construct with functions from $L^{\infty}([1, \infty))$ (resp. $\left.L^{\infty}([0, \infty))\right)$ replaced by continuous functions from $C_{b}([1, \infty)$ ) (resp. $\left.C_{b}([0, \infty))\right)$.

Let $\lceil x\rceil, x \geq 0$, denote the ceiling function. For $j \in \mathbb{N}$ define the maps $\ell^{\infty} \rightarrow \ell^{\infty}$ by

$$
\begin{aligned}
& T_{j}\left(\left\{a_{k}\right\}_{k=1}^{\infty}\right):=\left\{a_{k+j}\right\}_{k=1}^{\infty}, \\
& D_{j}\left(\left\{a_{k}\right\}_{k=1}^{\infty}\right):=\left\{a_{\left\lceil j^{-1} k\right\rceil}\right\}_{k=1}^{\infty}, \\
& C\left(\left\{a_{k}\right\}_{k=1}^{\infty}\right):=\left\{\frac{1}{n} \sum_{k=1}^{n} a_{k}\right\}_{n=1}^{\infty} .
\end{aligned}
$$

Define subsets of $S_{\infty}\left(\ell^{\infty}\right)$ by

$$
\begin{aligned}
& B L:=\left\{\omega \in S\left(\ell^{\infty}\right) \mid \omega \circ T_{j}=\omega \forall j \in \mathbb{N}\right\}, \\
& C B L:=\left\{\omega \in S\left(\ell^{\infty}\right) \mid \omega=\sigma \circ C, \sigma \in S_{\infty}\left(\ell^{\infty}\right)\right\}, \\
& B(C):=\left\{\omega \in S\left(\ell^{\infty}\right) \mid \omega \circ C=\omega\right\}, \\
& D_{2}:=\left\{\omega \in S_{\infty}\left(\ell^{\infty}\right) \mid\left(\omega \circ D_{2}-\omega\right)\left(\alpha\left(\left\{\mu_{n}(T)\right\}_{n=1}^{\infty}\right)\right)=0 \forall T \in \mathcal{M}_{1, \infty}\right\}, \\
& D L:=\left\{\omega \in S\left(\ell^{\infty}\right) \mid \omega \circ D_{j}=\omega \forall j \in \mathbb{N}\right\},
\end{aligned}
$$

where $\alpha$ is from (2.1). The set $B L$ is the classical set of Banach limits [23, 24]. The elements of $C B L$ are called Cesàro-Banach limits and the elements of $B(C)$ are called Cesàro invariant Banach limits. We note that (as the names suggest)

$$
B(C) \subsetneq C B L \subsetneq B L
$$

and

$$
D L \subsetneq D_{2} .
$$


For $a>0$ define the maps $L^{\infty}([0, \infty)) \rightarrow L^{\infty}([0, \infty))$ by

$$
\begin{aligned}
T_{a}(f)(t) & :=f(t+a), \\
D_{a}(f)(t) & :=f\left(a^{-1} t\right), \\
P_{a}(f)(t) & :=f\left(t^{a}\right), \\
C(f)(t) & :=\frac{1}{t} \int_{0}^{t} f(s) d s .
\end{aligned}
$$

Define $L^{-1}: L^{\infty}([1, \infty)) \rightarrow L^{\infty}([0, \infty))$ by

$$
L^{-1}(g)(t):=g\left(e^{t}\right)
$$

and, if $G: L^{\infty}([0, \infty)) \rightarrow L^{\infty}([0, \infty))$, set

$$
L(G): \quad L^{\infty}([1, \infty)) \rightarrow L^{\infty}([1, \infty)), \quad G \mapsto L \circ G \circ L^{-1}
$$

Similarly, if $\sigma \in S([0, \infty))$ set

$$
L(\sigma) \in S([1, \infty)), \quad \sigma \mapsto \sigma \circ L^{-1} .
$$

These definitions imply $L(\sigma) \circ L(G)=L(\sigma \circ G)$. It is known $L\left(T_{a}\right)=D_{e^{-a}}$ and $L\left(D_{a}\right)=P_{a^{-1}}$, see $[25, \S 1.1]$. Define subsets of $S_{\infty}([0, \infty))$ and $S_{\infty}([1, \infty))$ by

$$
\begin{aligned}
& B L[0, \infty):=\left\{\omega \in S([0, \infty)) \mid \omega \circ T_{a}=\omega \forall a>0\right\}, \\
& C B L[0, \infty):=\left\{\omega \in S([0, \infty)) \mid \omega=\sigma \circ C, \sigma \in S_{\infty}([0, \infty))\right\}, \\
& B(C)[0, \infty):=\{\omega \in S([0, \infty)) \mid \omega \circ C=\omega\}, \\
& D L[0, \infty):=\left\{\omega \in S([0, \infty)) \mid \omega \circ D_{a}=\omega \forall a>0\right\}, \\
& D L[1, \infty):=\{L(\omega) \mid \omega \in B L[0, \infty)\}, \\
& C D L[1, \infty):=\{L(\omega) \mid \omega \in C B L([0, \infty))\}, \\
& D(C)[1, \infty):=\{L(\omega) \mid \omega \in B(C)([0, \infty))\}, \\
& P D L[1, \infty):=\{L(\omega) \mid \omega \in B L[0, \infty) \cap D L[0, \infty)\}, \\
& M D L[1, \infty):=\{L(\omega) \mid \omega \in B L[0, \infty) \cap D L[0, \infty) \cap B(C)[0, \infty)\} .
\end{aligned}
$$

A subscript of $c$ for any of the sets (2.21)-(2.29) denotes the replacement of $L^{\infty}$-functions by $C_{b^{-}}$ functions, i.e. $B L_{c}[0, \infty)$ denotes the translation invariant states on the $C^{*}$-algebra $C_{b}([0, \infty))$ and it is a subset of $S C_{\infty}([0, \infty))$.

We now define the Dixmier traces corresponding to the singular states (2.8)-(2.11), (2.21)(2.29) and subscript $c$ versions.

Let $g^{*}$ be the monotonically decreasing left-continuous rearrangement of $|g|$, where $g \in$ $L^{\infty}([0, \infty))[26]$. Define

$$
\alpha_{t}\left(g^{*}\right):=\frac{1}{\log (1+t)} \int_{1}^{t} g^{*}(s) d s, \quad t \geq 1
$$

(the continuous version of (2.1)). Define the floor map $p$ from $\ell^{\infty}$ to bounded Borel functions on $[0, \infty)($ or $[1, \infty))$ by

$$
p\left(\left\{a_{k}\right\}_{k=1}^{\infty}\right)(t):=\sum_{k=1}^{\infty} a_{k} \chi_{[k, k+1)}(t), \quad t \geq 0 \quad(\text { or } 1)
$$


and the restriction map $r$ from bounded Borel functions on $[0, \infty)($ or $[1, \infty))$ to $\ell^{\infty}$

$$
r(f)_{n}:=f(n), \quad n \in \mathbb{N} .
$$

We have the following sets of unitarily invariant weights on $\mathcal{M}_{1, \infty}$ :

$$
\begin{aligned}
& \mathcal{V}_{B}=\left\{\operatorname{tr}_{\omega}(T):=\omega \circ \alpha_{t} \circ p\left(\left\{\mu_{n}(T)\right\}\right), T>0 \mid \omega \in B\right\}, \\
& B=\left\{\begin{array}{l}
D L[1, \infty), D L_{c}[1, \infty), \\
C D L[1, \infty), C D L_{c}[1, \infty), \\
D(C)[1, \infty), D(C)_{c}[1, \infty), \\
P D L[1, \infty), M D L[1, \infty)
\end{array}\right. \\
& \mathcal{V}_{B}=\left\{\operatorname{Tr}_{\omega}(T):=\omega \circ r \circ \alpha_{t} \circ p\left(\left\{\mu_{n}(T)\right\}\right), T>0 \mid \omega \in B\right\}, \quad B=D_{2}, \\
& \mathcal{W}_{Y}=\left\{\mathrm{f}_{\omega}(T):=\omega \circ \alpha_{e^{t}} \circ p\left(\left\{\mu_{n}(T)\right\}\right), T>0 \mid \omega \in Y\right\} \text {, } \\
& Y=\left\{\begin{array}{l}
B L[0, \infty), B L_{c}[0, \infty), \\
C B L[0, \infty), C B L_{c}[0, \infty), \\
B(C)[0, \infty), B(C)_{c}[0, \infty)
\end{array}\right. \\
& \mathcal{W}_{Y}=\left\{\mathrm{F}_{\omega}(T):=\omega \circ r \circ \alpha_{e^{t}} \circ p\left(\left\{\mu_{n}(T)\right\}\right), T>0 \mid \omega \in Y\right\}, \quad Y=B L, C B L, B(C) .
\end{aligned}
$$

By linear extension each of the unitarily invariant weights defines a positive trace with domain $\mathcal{M}_{1, \infty}$.

The reader should rightly be bewildered by this variety of Dixmier traces. Before we explain the origin of so many options, let us clarify the picture. From Appendix A we have

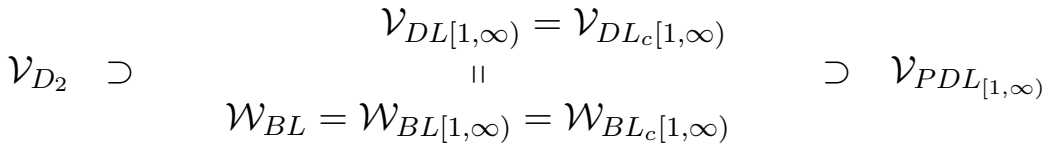

$$
\begin{aligned}
& \mathcal{V}_{C D L[1, \infty)}=\mathcal{V}_{C D L_{c}[1, \infty)} \\
& \text { II } \\
& \mathcal{W}_{C B L}=\mathcal{W}_{C B L[1, \infty)}=\mathcal{W}_{C B L_{c}[1, \infty)} \\
& \text { U } \\
& \mathcal{V}_{D(C)[1, \infty)}=\mathcal{V}_{D(C)_{c}[1, \infty)} \\
& \mathcal{W}_{B(C)}=\mathcal{W}_{B(C)[1, \infty)}=\mathcal{W}_{B(C)_{c}[1, \infty)} \quad \supset \quad \mathcal{V}_{M D L_{[1, \infty)}}
\end{aligned}
$$

Why did such a variety result? Connes' trace theorem [17] used the generalised limits originally specified by Dixmier [22]. Connes later introduced Dixmier traces defined by the sets $C D L_{c}[1, \infty)$ [2, pp. 303-308], after explaining [2, p. 305], following Dixmier [22], that any $\omega \in D_{2}$ was sufficient to define a Dixmier trace. In the same location [2, p. 308] Connes introduced the idea of measurability associated to the set of traces defined by $C D L_{c}[1, \infty$ ) (see the next section).

Translation invariance in Dixmier's original specification is redundant, hence the set of functionals $\mathcal{V}_{D L[1, \infty)}$ are generally termed the (original) Dixmier traces, while the more restrictive set $\mathcal{V}_{C D L_{c}[1, \infty)}$ we termed Connes-Dixmier traces, in [27], to distinguish them. The text [27] also initiated the identifications in (2.37), where our aim was to identify Dixmier and ConnesDixmier traces with subsets of singular symmetric functionals $[28,29,30]$, i.e. $\mathcal{V}_{D L[1, \infty)}=\mathcal{W}_{B L}$ and $\mathcal{V}_{C D L_{c}[1, \infty)}=\mathcal{W}_{C B L}$. The form of the functionals $\mathcal{W}_{B L, C B L}$ is simpler than it looks in (2.36). 
Indeed, as a result of [27] and [29], one can write any Dixmier trace (up to a constant) as

$$
\mathrm{F}_{\omega}(T)=\omega\left(\left\{\frac{1}{N} \sum_{k=1}^{2^{N}} \mu_{k}(T)\right\}_{N=1}^{\infty}\right), \quad T>0
$$

for some Banach limit $\omega$, and any Connes-Dixmier trace using the same formula except with a Cesàro-Banach limit $\omega \in C B L$.

The sets $\mathcal{V}_{D(C)[1, \infty)}$ and $\mathcal{V}_{M D L[1, \infty)}$ were introduced with a different intention. The paper [25] by A. Carey, J. Phillips, and the second author, demonstrated the residue and heat kernel formulations of the Dixmier trace, and did so with the type I von Neumann algebra $(\mathcal{L}(H)$, $\operatorname{Tr})$ replaced with any semifinite von Neumann algebra $(\mathcal{N}, \tau)$ with faithful normal semifinite trace $\tau$, see also $[19,31,16]$. The generalised $s$-numbers $\rho_{s}(T)$ of a $\tau$-compact operator $T$ in $\mathcal{N}$, [8], provide a generalisation of singular values, such that $\rho_{s}(T)=p\left(\left\{\mu_{n}(T)\right\}_{m=1}^{\infty}\right)$ for the pair $(\mathcal{L}(H), \operatorname{Tr})$. To use the generalised $s$-numbers, one replaces the sequence (2.1) by the function (2.30). For the semifinite pair $(\mathcal{N}, \tau)$ only the functionals $\mathcal{V}_{D L[1, \infty)}$ (and all continuous variants in (2.33) and (2.35)) can be defined.

The set $M D L[1, \infty)$, which is non-empty [25, p. 74], was a constraint used in [25] to identify the Dixmier trace with residues of a zeta function and heat kernel asymptotics (see Section 5 below). Later results on zeta functions [15] enabled a residue formulation for all Dixmier traces in the set $\mathcal{V}_{P D L[1, \infty)}$. It is still open whether a residue formulation exists for all functionals in the set $\mathcal{V}_{D L[1, \infty)}$. While residue formulations were able to drop the Cesàro invariance condition (which is unnecessary for the weak*-Karamata theorem [15, p. 271]), a later heat kernel formulation due to A. Sedaev dropped the power invariance requirement (as [15, Proposition 4.3] is unnecessary for the heat kernel). In [32], heat kernel formulations were derived for Connes-Dixmier traces in the sets $\mathcal{V}_{C D L_{c}[1, \infty)}$ and $\mathcal{V}_{D(C)_{c}[1, \infty)}$.

The set $D(C)[1, \infty)$ was used in $[16, \S 1.3]$. Finally, the set $B(C)$ was introduced in the paper [34] with an application to measurability (see the next section).

It will be convenient to simplify the schematic (2.37) of Dixmier traces. Set

$$
\begin{aligned}
& \mathcal{V}_{\frac{1}{2}}:=\mathcal{V}_{D_{2}}, \\
& \mathcal{V}_{1}:=\mathcal{V}_{D L[1, \infty)}=\mathcal{V}_{D L_{c}[1, \infty)}=\mathcal{W}_{B L_{c}[1, \infty)}=\mathcal{W}_{B L[1, \infty)}=\mathcal{W}_{B L}, \\
& \mathcal{V}_{2}:=\mathcal{V}_{C D L[1, \infty)}=\mathcal{V}_{C D L_{c}[1, \infty)}=\mathcal{W}_{C B L_{c}[1, \infty)}=\mathcal{W}_{C B L[1, \infty)}=\mathcal{W}_{C B L}, \\
& \mathcal{V}_{3}:=\mathcal{V}_{D(C)[1, \infty)}=\mathcal{V}_{D(C)_{c}[1, \infty)}=\mathcal{W}_{B(C)_{c}[1, \infty)}=\mathcal{W}_{B(C)[1, \infty)}=\mathcal{W}_{B(C)}
\end{aligned}
$$

The schematic (2.37) is then simplied to Dixmier traces $\left(\mathcal{V}_{1}\right)$, Connes-Dixmier traces $\left(\mathcal{V}_{2}\right)$ and Cesàro invariant Dixmier traces $\left(\mathcal{V}_{3}\right)$ :

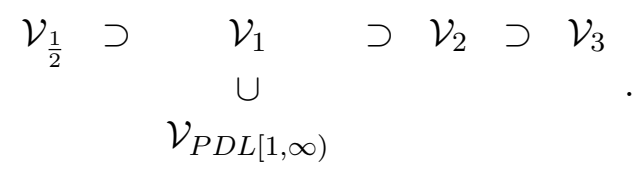

It is known $\mathcal{W}_{B(C)} \subsetneq \mathcal{W}_{C B L} \subsetneq \mathcal{W}_{B L}$, which is not trivial, see [27] and [34]. Hence the inclusions are strict, i.e. $\mathcal{V}_{1} \supsetneq \mathcal{V}_{2} \supsetneq \mathcal{V}_{3}$. We do not know whether $\mathcal{V}_{\frac{1}{2}} \supset \mathcal{V}_{1}$ is strict, i.e. anything is gained from the larger set of generalised limits. In some NCG papers Dixmier traces are defined with vague reference to the type of generalised limit being used, relying on the assumption of measurability (next section) to make the distinction irrelevant. To our knowledge, the classes of functionals in (2.42) represent the known relevant distinctions in measuring the 'log divergence of the trace' using the idea of Dixmier. 


\subsection{Singular symmetric functionals}

It would be remiss to finish a section on Dixmier traces without mentioning singular symmetric functionals.

Consider the following well-known formula for the canonical trace, a special case of the Lidskii formula ${ }^{3}$ :

$$
\operatorname{Tr}(T)=\lim _{N \rightarrow \infty} \sum_{k=1}^{N} \mu_{k}(T), \quad T>0,
$$

which defines a weight that is finite on the ideal $\mathcal{L}^{1}$ of trace class operators. The formula (2.43) makes the positivity and trace property evident (recall $\mu_{k}\left(U^{*} T U\right)=\mu_{k}(T)$ for all unitaries $U \in \mathcal{L}(H)$ and $\left.T \in \mathcal{L}^{\infty}\right)$, and makes it easy to see $S \mapsto \operatorname{Tr}(S T), S \in \mathcal{L}(H), T \in \mathcal{L}^{1}$, is a positive continuous linear functional on $\mathcal{L}(H)$ by the same arguments as for the formula (2.3).

Linearity is not immediately evident from (2.43) as it was not for (2.3) (but is evident from the definition of the trace $\operatorname{Tr}(T)=\sum_{m}\left\langle h_{m} \mid T h_{m}\right\rangle,\left\{h_{m}\right\}_{m=1}^{\infty}$ an orthonormal basis of $H$, which will be of relevance in Section 6).

Both formula (2.43) and (2.3) have the general format

$$
f\left(\left\{\mu_{n}(T)\right\}\right),
$$

where $f$ is a positive linear functional on the sequence space $\ell^{1}$ in the case of $\operatorname{Tr}$, and the sequence space

$$
m_{1, \infty}:=\left\{x=\left\{x_{n}\right\}_{n=1}^{\infty} \in \ell^{\infty} \mid \sup _{k} \alpha\left(x^{*}\right)_{k}<\infty\right\}
$$

where $\alpha$ is the sequence from (2.1) and $x^{*}$ is the sequence $\left\{\left|x_{n}\right|\right\}_{n=1}^{\infty}$ rearranged in nonincreasing order, in the case of $\operatorname{Tr}_{\omega}$.

A Calkin sequence space $j$ is a subset of $c_{0}$ such that $x \in j$ and $y^{*} \leq x^{*}$ implies $y \in j$ (called the Calkin property) [11, p. 18]. It is known that a subset $\mathcal{J}$ of compact operators is a two-sided ideal if and only if the sequence space $j_{\mathcal{J}}=\left\{x \in c_{0} \mid x^{*}=\left\{\mu_{n}(T)\right\}_{n=1}^{\infty}, T \in \mathcal{J}\right\}$ is a Calkin space [37].

A symmetric sequence space is a Calkin space $e$ with norm $\|\cdot\|_{e}$ such that $y^{*} \leq x^{*}$ implies $\|y\|_{e} \leq\|x\|_{e} \forall x \in e$. A symmetric ideal of compact operators $\mathcal{E}$ is a two sided ideal with a norm $\|\cdot\|_{\mathcal{E}}$ such that $\|S T V\|_{\mathcal{E}} \leq\|S\|\|T\|_{\mathcal{E}}\|V\|, \forall S, V \in \mathcal{L}(H), T \in \mathcal{E}$. It is known (actually, only recently when [38] extended the finite dimensional result of J. von Neumann in [12]) that $\mathcal{E}$ is a symmetric ideal if and only if $j_{\mathcal{E}}$ is a symmetric sequence space and that $\|T\|_{\mathcal{E}}=\left\|\left\{\mu_{n}(T)\right\}_{n=1}^{\infty}\right\|_{j_{\epsilon}}$. For example $\ell^{1}$ (resp. $m_{1, \infty}$ ) is the symmetric sequence space associated to the symmetric ideal $\mathcal{L}^{1}$ (resp. $\mathcal{M}_{1, \infty}$ ).

These correspondences allow us to form the equation (2.44) for any symmetric ideal $\mathcal{E}$ and using $0<f \in j_{\mathcal{E}}^{*}$. The functional defined on $\mathcal{E}_{+}$by (2.44) is positive, unitarily invariant, continuous (in the symmetric norm $\|\cdot\|_{\mathcal{E}}$ ), but not necessarily linear. To obtain linearity, and so derive a trace on $\mathcal{E}$, one introduces the notions of symmetric and fully symmetric functionals. If $e$ is a symmetric sequence space, then a functional $0<f \in e^{*}$ is called symmetric if $y^{*} \leq x^{*}$ implies $f\left(y^{*}\right) \leq f\left(x^{*}\right)$. If $x, y \in e$, we write $y \prec \prec x$ (and say $y$ is submajorised by $x$ ) when

$$
\sum_{n=1}^{N} y_{n}^{*} \leq \sum_{n=1}^{N} x_{n}^{*} \quad \forall N \in \mathbb{N} .
$$

\footnotetext{
${ }^{3}$ There are Lidksii type formulations for the Dixmier trace, which for sake of brevity we will not discuss in this text, see [35] and [33]. For spectral asymptotic formulae see [36, 16].
} 
A symmetric sequence space $e$ is called fully symmetric if $x \in e$ and $y \prec \prec x$ implies $y \in e$ (the Calkin property with rearrangment order replaced by Hardy-Littlewood-Pólya submajorisation [39]). A functional $0<f \in e^{*}$ on a fully symmetric sequence space $e$ is called fully symmetric if $y \prec \prec x$ implies $f\left(y^{*}\right) \leq f\left(x^{*}\right)$. Both $\ell^{1}$ and $m_{1, \infty}$ are fully symmetric sequence spaces. The ideal $\mathcal{E}$ is called fully symmetric if $j_{\mathcal{E}}$ is a fully symmetric sequence space. Finally, a symmetric functional $0<f \in e^{*}$ is called singular if $f$ vanishes on all finite sequences.

In $[28,29,30]$ and $[40, \text { p. } 77]^{4}$, it was shown (singular) symmetric linear functionals $0<f \in j_{\mathcal{E}}^{*}$ correspond to (singular) positive linear unitarily invariant functionals on fully symmetric ideals $\mathcal{E}$ using the formula (2.44).

Now, the relevant question is whether all Dixmier traces $\left(\mathcal{V}_{1}\right)$ on $\mathcal{M}_{1, \infty}$ are constructed from singular symmetric functionals $0<f \in m_{1, \infty}^{*}$. The answer is yes. In fact, we now know $f\left(\left\{\mu_{n}(T)\right\}\right)$ defines a Dixmier trace if and only if $0<f \in m_{1, \infty}^{*}$ is a singular fully symmetric functional (a result of the second author, N. Kalton and A. Sedaev, to appear). (Fully) symmetric singular functionals on (fully) symmetric ideals thus generalise the construction of Dixmier (other generalisations exist, see [31,41]). Another relevant question is whether all positive linear unitarily invariant singular functionals on $\mathcal{M}_{1, \infty}$ constructed from singular symmetric functionals are Dixmier traces. The answer is no. There are singular symmetric functionals $0<f \in m_{1, \infty}^{*}$ which are not fully symmetric, yet $f\left(\left\{\mu_{n}(T)\right\}\right)$ defines a singular positive linear unitarily invariant functional on $\mathcal{M}_{1, \infty}[40$, p. 79], i.e. we now know

there exist singular traces $0<\rho \in \mathcal{M}_{1, \infty}^{*}$ that are not Dixmier traces.

In particular, [40, p. 79] informs us that there exists a non-trivial symmetric functional $0<f_{p} \in$ $m_{1, \infty}^{*}$ such that $f_{p}\left(\left\{\frac{1}{n}\right\}_{n=1}^{\infty}\right)=0$ for the harmonic sequence $\left\{\frac{1}{n}\right\}_{n=1}^{\infty} \in m_{1, \infty}$ (and hence it is not fully symmetric $\left.{ }^{5}\right)$. Setting $f=f_{1}+f_{p}$, where $f_{1}$ is any symmetric functional on $m_{1, \infty}$ for which $f_{1}\left(\left\{\frac{1}{n}\right\}_{n=1}^{\infty}\right)=1$, yields a symmetric functional which cannot be fully symmetric. The positive singular trace $\rho$ defined by $\rho(T)=\left(f_{1}+f_{p}\right)\left(\left\{\mu_{n}(T)\right\}\right)$ is not a Dixmier trace, yet $\rho(T)=1$ for any positive compact operator $T$ with singular values given by the harmonic sequence (such as the inverse of the square root of the Laplacian on the 1 -torus $\mathbb{R} / \mathbb{Z}$ ).

\section{Measurability}

Measurability, as defined by Connes [2, p. 308], is the notion that

$$
\operatorname{Tr}_{\omega}(T)=\operatorname{Tr}_{\omega^{\prime}}(T) \quad \forall \omega, \omega^{\prime} \in B,
$$

where $B$ is some subset of generalised limits which defines a Dixmier trace (see the previous section). Specifically, Connes used the set $B=C D L_{c}[1, \infty)[2$, p. 305]. A compact operator $T \in \mathcal{M}_{1, \infty}$ satisfying (3.1) is called $B$-measurable.

With reference to the noncommutative integral (1.1), one desires that the products $a\langle D\rangle^{-n}$ are $(B$-)measurable, so that the formula $(1.1)$ is independent of the generalised limit used. That the projections in $\mathcal{A}^{\prime \prime}$ (using the extension (1.6)) have a unique value corresponding to the 'infinitesimal' $\langle D\rangle^{-n}$ parallels the idea that the Lebesgue measurable sets are those with a unique 'size' based on the metric.

Define the Tauberian operators

$$
\mathcal{T}^{+}:=\left\{0<T \in \mathcal{M}_{1, \infty} \mid\left\{\frac{1}{\log (1+k)} \sum_{n=1}^{k} \mu_{n}(T)\right\}_{k=1}^{\infty} \in c\right\} .
$$

\footnotetext{
${ }^{4}$ These texts called symmetric (resp. rearrangement invariant) the functionals which are here called fully symmetric (resp. symmetric).

${ }^{5}$ All sequences in the unit ball of $m_{1, \infty}$ are submajorised by the harmonic sequence. A non-trivial continuous functional on $m_{1, \infty}$ that vanishes on the harmonic sequence is not fully symmetric, else non-triviality is contradicted.
} 
By construction, for a subset of generalised limits $B \subset S_{\infty}\left(\ell^{\infty}\right)$,

$$
\operatorname{Tr}_{\omega}(T)=\lim _{k \rightarrow \infty} \frac{1}{\log (1+k)} \sum_{n=1}^{k} \mu_{n}(T) \quad \forall \omega \in B, \quad T \in \mathcal{T}^{+} .
$$

Hence the Tauberian operators are $B$-measurable for any $B \subset S_{\infty}\left(\ell^{\infty}\right)$. We know from (2.42) in the previous section there are three (possibly five) main varieties of Dixmier trace. Then, a priori, there are three (possibly five) versions of measurability. Define

$$
\mathcal{K}_{i}:=\left\{T \in \mathcal{M}_{1, \infty} \mid \mathrm{f}(T)=\text { const } \forall \mathrm{f} \in \mathcal{V}_{i}\right\}, \quad i=\frac{1}{2}, 1,2,3, P D L[1, \infty)
$$

and let $\mathcal{K}_{i}^{+}$denote $0<T \in \mathcal{K}_{i}$. We concentrate on positive operators for the moment. Then, from (2.42), we know that

$$
\begin{array}{cccccc}
\mathcal{T}^{+} \subset \mathcal{K}_{\frac{1}{2}}^{+} & \subset & \mathcal{K}_{1}^{+} \quad \subset \mathcal{K}_{2}^{+} \subset \mathcal{K}_{3}^{+} \\
& & \\
& & \mathcal{K}_{P D L[1, \infty)}^{+} \\
& &
\end{array}
$$

where the inclusions may or may not be strict. In [27], our main result was that $\mathcal{K}_{2}^{+}=\mathcal{T}^{+}$, identifying Connes' definition of measurability in [2] for positive operators with the Tauberian operators. Specifically we introduced the notion of the Cesàro limit property [27, p. 97] and any subset of Banach limits $B$ satisfying this property has $B$-measurable positive operators (using the equivalence $\mathcal{V}_{D L[1, \infty)}=\mathcal{W}_{B L}$ ) equivalent to Tauberian operators. The set $C B L$ of CesàroBanach limits satisfy the Cesàro limit property. From the paper of E. Semenov and the second author [34], we now know that $\mathcal{K}_{3}^{+} \neq \mathcal{T}^{+}$. From this, the schematic (3.4) can be reduced to

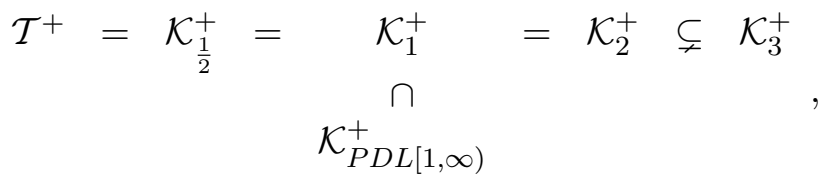

so that the set of positive measurable operators according to Connes-Dixmier traces (and any larger set of Dixmier traces) is equivalent to the Tauberian operators, but the set of positive measurable operators for Cesàro invariant Dixmier traces is strictly larger than the Tauberian operators. The Cesàro limit property is sufficient for equality with the Tauberian operators, it is an open question as to whether the property is a necessary condition (the set $B(C)$ of Cesàro invariant Banach limits does not satisfy the property and $\left.\mathcal{K}_{3}^{+} \neq \mathcal{T}^{+}\right)$. Whether $\mathcal{K}_{P D L[1, \infty)}^{+}$is equal to the Tauberian operators is also an open question.

It is interesting to note that some notions of measurability can exclude the Tauberian operators. From the last section on symmetric functionals, since the set of Dixmier traces corresponds to the set of fully symmetric functionals $0<f \in m_{1, \infty}^{*}$, the Tauberian operators $\mathcal{T}^{+}$are the set of $0<T \in \mathcal{M}_{1, \infty}$ such that the (2.44) is independent of the fully symmetric functional $f$. Given (2.45), we could try to widen this notion. For example, let $\mathcal{V}_{\mathrm{sym}}^{+}$to be the set of all positive singular traces $\rho$ on $\mathcal{M}_{1, \infty}$ such that $\rho(T)=f\left(\left\{\mu_{n}(T)\right\}\right) \forall T>0$ for some symmetric functional $0<f \in m_{1, \infty}^{*}$. Then set

$$
\mathcal{K}_{\text {sym }}^{+}:=\left\{0<T \in \mathcal{M}_{1, \infty} \mid \rho(T)=\text { const } \forall \rho \in \mathcal{V}_{\text {sym }}^{+}\right\} .
$$

We are informed by [40, p. 79] that $\mathcal{T}^{+} \not \subset \mathcal{K}_{\text {sym }}^{+}$. So, widening the notion of measurable in this manner excludes some Tauberian operators. We are indirectly informed by this result that there exist singular traces on $\mathcal{M}_{1, \infty}$ that do not arise from a generalised limiting procedure applied to the sequence $\left\{\frac{1}{\log (1+n)} \sum_{k=1}^{n} \mu_{k}(T)\right\}_{n=1}^{\infty}$. 
In summary, the best way to avoid difficulties with the notion of measurability when one is using Dixmier traces to define the noncommutative integral (1.1) is to have the Tauberian condition:

$$
a^{\frac{1}{2}}\langle D\rangle^{-n} a^{\frac{1}{2}} \in \mathcal{T}^{+}, \quad \forall 0<a \in \mathcal{A} .
$$

However, we know of no general criteria involving the $*$-algebra $\mathcal{A}$ and the selfadjoint operator $D$ that demonstrates (3.6) besides direct examination of singular values. Finding such a criteria is an open problem. The linear span $\mathcal{T}$ of $\mathcal{T}^{+}$is not an ideal of compact operators, indeed we know $\mathcal{K}_{i}, i=1,2,3$, is not an ideal for any class of Dixmier trace.

There are specific situations where (3.6) is implied without having to check singular values. The functionals defined by (1.1) are all traces on the unital $*$-algebra $\mathcal{A}$. If $\langle D\rangle^{-n}$ is a Tauberian operator and $\operatorname{Tr}_{\omega}\left(\langle D\rangle^{-n}\right)>0$, then the traces from (1.1) can be normalised by the same constant independent of $\omega$,

$$
\frac{\operatorname{Tr}_{\omega}\left(a\langle D\rangle^{-n}\right)}{\operatorname{Tr}_{\omega}\left(\langle D\rangle^{-n}\right)} .
$$

A unique trace state on $\mathcal{A}$ automatically implies the trace states in (3.7) have the same value for all $\omega \in D_{2}$. Hence, for all $a>0, a^{1 / 2}\langle D\rangle^{-n} a^{1 / 2} \in \mathcal{T}^{+}$and condition (3.6) is satisfied. The principle of a unique (singular) trace is also the construct for measurability of pseudo-differential operators of order $-n$. So far as we know, these situations of unique trace, besides very strong geometric conditions involving Hochschild cohomology and axioms for noncommutative manifolds [2, p. 309], [3, p. 160] provide the only handle on (3.6).

The unsatisfactory feature of this situation, from a measure-theoretic point of view, is that a unique trace often enables more general traces than Dixmier traces on compact operators to be used in (1.1) to obtain the same result (see the sections below). Where is the definitive rationale that Dixmier traces should solely appear in connection with the noncommutative integral?

\subsection{Decomposition of measurable operators}

We concentrated in the previous section on identifying those positive compact operators for which $\operatorname{Tr}_{\omega}(T)=$ const for various $\operatorname{Tr}_{\omega}$ in the classes $\mathcal{V}_{1}, \mathcal{V}_{2}, \mathcal{V}_{3}$. We know that general $T \in \mathcal{M}_{1, \infty}$ polarise, i.e.

$$
T=T_{1}-T_{2}+i T_{3}-i T_{4}, \quad 0<T_{j} \in \mathcal{M}_{1, \infty}, \quad j=1,2,3,4,
$$

and that

$$
\operatorname{Tr}_{\omega}(T)=\omega\left(\left\{\frac{1}{\log (1+N)} \sum_{k=1}^{N} \mu_{k}\left(T_{1}\right)-\mu_{k}\left(T_{2}\right)+i \mu_{k}\left(T_{3}\right)-i \mu_{k}\left(T_{4}\right)\right\}_{k=1}^{\infty}\right) .
$$

For convenience, denote

$$
\tilde{\mu}_{k}(T):=\mu_{k}\left(T_{1}\right)-\mu_{k}\left(T_{2}\right)+i \mu_{k}\left(T_{3}\right)-i \mu_{k}\left(T_{4}\right), \quad T \in \mathcal{L}^{\infty}(H) .
$$

It is evident if $T \in \mathcal{T}:=\operatorname{span}_{\mathbb{C}}\left(\mathcal{T}^{+}\right)$(i.e. $T_{1}, T_{2}, T_{3}, T_{4} \in \mathcal{T}^{+}$), or

$$
T \in \tilde{\mathcal{T}}:=\left\{T \in \mathcal{M}_{1, \infty} \mid\left\{\frac{1}{\log (1+N)} \sum_{k=1}^{N} \tilde{\mu}_{k}(T)\right\}_{k=1}^{\infty} \in c\right\} \supset \mathcal{T},
$$

then

$$
\operatorname{Tr}_{\omega}(T)=\lim _{N \rightarrow \infty} \frac{1}{\log (1+N)} \sum_{k=1}^{N} \tilde{\mu}_{k}(T) .
$$


Hence $\operatorname{Tr}_{\omega}(T)=$ const for $T \in \tilde{\mathcal{T}}$. Does the reverse implication hold for the various classes $\operatorname{Tr}_{\omega} \in \mathcal{V}_{1}, \mathcal{V}_{2}, \mathcal{V}_{3}$ ?

Recent work by the second author, N. Kalton, and A. Sedaev, to appear, indicates that the proposition

$$
\mathrm{f}(T)=\text { const } \forall \mathrm{f} \in \mathcal{V}_{1} \Rightarrow\left\{\frac{1}{\log (1+N)} \sum_{k=1}^{N} \tilde{\mu}_{k}(T)\right\}_{k=1}^{\infty} \in c
$$

is false. In the next section on symmetric subideals we briefly describe the strictness of the inclusions in the following schematic:

$$
\tilde{\mathcal{T}} \subsetneq \mathcal{K}_{1} \subsetneq \mathcal{K}_{2} \subsetneq \mathcal{K}_{3}
$$

The place of $\mathcal{K}_{\frac{1}{2}}$ (and $\left.\mathcal{K}_{P D L[1, \infty)}\right)$ is open.

The strictness of the inclusion $\tilde{\mathcal{T}} \subsetneq \mathcal{K}_{1}$ and the result (3.5) for positive elements implies

$$
\operatorname{span}_{\mathbb{C}}\left(\mathcal{K}_{i}^{+}\right) \subsetneq \mathcal{K}_{i}, \quad i=1,2 .
$$

It is evident from $\mathrm{f}\left(T^{*}\right)=\overline{\mathrm{f}(T)}, \mathrm{f} \in \mathcal{V}_{i}, i=\frac{1}{2}, 1,2, P D L[1, \infty)$, that the sets $\mathcal{K}_{i}$ are closed under the $*$-operation. It follows that $T \in \mathcal{K}_{i} \nRightarrow|T| \in \mathcal{K}_{i}, i=1,2$.

As a consequence, we lack a basic decomposition result of integration theory. Suppose $T=T^{*}$ with the standard unique decomposition into positive and negative operators

$$
T=T_{+}-T_{-}, \quad T_{ \pm}:=\frac{1}{2}(|T| \pm T) .
$$

Then,

$$
T=T^{*} \in \mathcal{K}_{i} \nRightarrow T_{+}, T_{-} \in \mathcal{K}_{i}, \quad i=1,2 .
$$

Compare this with the result for a Borel measure space $(X, \mu)$ (also true for the noncommutative variant of $\tau$-measurable operators on $(\mathcal{N}, \tau)$ for a semifinite von Nuemann algebra $\mathcal{N}$ with faithful normal semifinite trace $\tau$ )

$$
f=\bar{f} \in L^{0}(X, \mu) \Rightarrow \exists ! 0<f_{+}, f_{-} \in L^{0}(X, \mu) \quad \text { s.t. } f=f_{+}-f_{-},
$$

where $L^{0}(X, \mu)$ denotes the set of (equivalence classes) of $\mu$-measurable functions on $X$.

\subsection{Closed symmetric subideals}

Define symmetric subspaces of the symmetric sequence space $m_{1, \infty}$ by

$$
\begin{aligned}
& u_{1, \infty}=\left\{x \in m_{1, \infty} \mid x^{*}(n)=o\left(\frac{\log n}{n}\right)\right\}, \\
& \ell^{1, w}=\left\{x \in m_{1, \infty} \mid x^{*}(n)=O\left(\frac{1}{n}\right)\right\} .
\end{aligned}
$$

The ideal of compact operators $\mathcal{U}_{1, \infty}$ and $\mathcal{L}^{1, w}$, defined by

$$
u_{1, \infty}=j_{\mathcal{U}_{1, \infty}}, \quad \ell^{1, w}=j_{\mathcal{L}^{1, w}}
$$

are quasi-Banach symmetric ideals. Notice also

$$
\mathcal{L}^{1, w} \subsetneq \mathcal{U}_{1, \infty} \subsetneq \mathcal{M}_{1, \infty}
$$


Define, as before,

$$
\tilde{\mathcal{T}}:=\left\{T \in \mathcal{M}_{1, \infty} \mid\left\{\frac{1}{\log (1+N)} \sum_{k=1}^{N} \tilde{\mu}_{k}(T)\right\}_{k=1}^{\infty} \in c\right\} .
$$

New results of N. Kalton, A. Sedaev, and the second author, state that

$$
\tilde{\mathcal{T}} \cap \mathcal{U}_{1, \infty}=\mathcal{K}_{1} \cap \mathcal{U}_{1, \infty}
$$

and that $\mathcal{U}_{1, \infty}$ is the maximal symmetric subideal $\mathcal{E} \subset \mathcal{M}_{1, \infty}$ for which (3.14) holds. Thus,

$$
\tilde{\mathcal{T}}=\tilde{\mathcal{T}} \cap \mathcal{M}_{1, \infty} \neq \mathcal{K}_{1} \cap \mathcal{M}_{1, \infty}=\mathcal{K}_{1}
$$

Their results also show

$$
\tilde{\mathcal{T}} \cap \mathcal{U}_{1, \infty} \neq \mathcal{K}_{2} \cap \mathcal{U}_{1, \infty}
$$

Hence $\mathcal{K}_{1} \neq \mathcal{K}_{2}$. It is an open question as to the existence of a maximal closed symmetric subideal $\mathcal{U}_{2}$ such that $\tilde{\mathcal{T}} \cap \mathcal{U}_{2}=\mathcal{K}_{2} \cap \mathcal{U}_{2}$. Is $\mathcal{L}^{1, w} \subseteq \mathcal{U}_{2}$ ?

\section{Origin of the noncommutative integral}

The origin of the formula (1.1) as the integral in noncommutative geometry lies in Connes' trace theorem [17, Theorem 1], [42, p. 293]:

Theorem 4.1 (Connes' trace theorem). Let $M$ be a compact $n$-dimensional manifold, $\mathcal{E}$ a complex vector bundle on $M$, and $P$ a pseudo-differential operator of order $-n$ acting on sections of $\mathcal{E}$. Then the corresponding operator $P$ in $H=L^{2}(M, \mathcal{E})$ belongs to $\mathcal{M}_{1, \infty}(H)$ and one has:

$$
\operatorname{Tr}_{\omega}(P)=\frac{1}{n} \operatorname{Res}(P)
$$

for any $\operatorname{Tr}_{\omega} \in \mathcal{V}_{1}$.

Here Res is the restriction of the Adler-Manin-Wodzicki residue to pseudo-differential operators of order $-n[43,17]$. The monograph $[42, \S 7]$ provides a detailed introduction to the residue Res and the origin and framework of the formula. We recall that, for classical pseudo-differential operators of order $-n$ acting on the trivial line bundle $\left(H=L^{2}(M)\right)$,

$$
\operatorname{Res}(P)=\frac{1}{(2 \pi)^{n}} \int_{S^{1} M} \sigma_{-n}(P)(x, \xi) d x d \xi,
$$

where $d x d \xi$ is the Liouville measure (volume form) on the cosphere bundle $S^{1} M$ (with fibres $S^{1} M_{x} \cong\{x\} \times S^{n-1}$ over $x \in M$ where $S^{n}$ is the unit $n$-sphere) and $\sigma_{-n}$ is the principal symbol of $P$. On the left hand side of (4.1) one should read for $P$ the unique extension of the negative order pseudo-differential operator $C^{\infty}(M) \rightarrow C^{\infty}(M)$ to a compact operator $L^{2}(M) \rightarrow$ $L^{2}(M)[44]$.

Let $\Delta$ denote the Hodge Laplacian on $M$ and $P_{a} \in B\left(L^{2}(M)\right)$ denote the extension of the zeroth order pseudo-differential operator associated to a function $a \in C^{\infty}\left(S^{1} M\right)$. Since

$$
\left.\sigma_{-n}\left(P_{a}(1+\Delta)^{-n / 2}\right)\right|_{S^{1} M}=a
$$

formula (4.1) provides

$$
\operatorname{Tr}_{\omega}\left(P_{a}(1+\Delta)^{-n / 2}\right)=\frac{1}{n(2 \pi)^{n}} \int_{S^{1} M} a(x, \xi) d x d \xi
$$


From the inclusion $a \in C^{\infty}(M) \hookrightarrow C^{\infty}\left(S^{1} M\right)$ the operator $P_{a}, a \in C^{\infty}(M)$, is the multiplier by the function $a \in C^{\infty}(M)$ and [42, Corollary 7.21], [16, $\left.\S 1.1\right]$ or [45, p. 98],

$$
\operatorname{Tr}_{\omega}\left(P_{a} T_{\Delta}\right)=\frac{\operatorname{Vol}\left(S^{n-1}\right)}{n(2 \pi)^{n}} \int_{M} a(x) d x, \quad a \in C^{\infty}(M),
$$

where we set

$$
T_{\Delta}:=(1+\Delta)^{-n / 2} \in \mathcal{M}_{1, \infty}
$$

Equation (4.3) originated the use of the term integral for the expression (1.1).

Why is a singular trace needed in formula (4.1)? By the asymptotic expansion of classical symbols $P-P_{0} \equiv \sigma_{-n}(P)$ for a classical pseudo-differential operator $P$ of order $-n$ where $P_{0}$ is of order $<-n$ and $\sigma_{-n}(P)$ is the principal symbol of $P$ [46, XVIII]. All pseudo-differential operators of order $<-n$ on a compact $n$-dimensional manifold have trace class extensions [44]. Hence any singular functional $\mathrm{f} \in \mathcal{M}_{1, \infty}$ applied to $P$, i.e. $P \mapsto \mathrm{f}(P)$, since it vanishes on $\mathcal{L}^{1}$, is equivalent to a functional on symbols of order $-n$. Moreover the vanishing on $\mathcal{L}^{1}$ permits that the statement need only be proved locally. Hence $M$ may be taken to be homogeneous. If $\mathrm{f}$ is a positive trace this functional on symbols of order $-n$ is equivalent to an invariant measure on $S^{1} M$. Hence, up to a constant, it identifies with the Liouville integral.

The above argument, from the proof of Connes' trace theorem in [17], implies that any positive singular trace $0<\rho \in \mathcal{M}_{1, \infty}^{*}$ can be substituted for the Dixmier trace in (4.1). Therefore we may write, using the above notation:

Theorem 4.2 (Connes' trace theorem). Let $M$ be a compact $n$-dimensional manifold, $\mathcal{E}$ a complex vector bundle on $M$, and $P$ a pseudo-differential operator of order $-n$ acting on sections of $\mathcal{E}$. Then the corresponding operator $P$ in $H=L^{2}(M, \mathcal{E})$ belongs to $\mathcal{M}_{1, \infty}(H)$ and one has:

$$
\rho(P)=\frac{\rho\left(T_{\Delta}\right)(2 \pi)^{n}}{\operatorname{Vol}\left(S^{1} M\right)} \operatorname{Res}(P)
$$

for any singular trace $0<\rho \in \mathcal{M}_{1, \infty}(H)^{*}$.

Singular traces $0<\rho \in \mathcal{M}_{1, \infty}\left(L^{2}(M, \mathcal{E})\right)^{*}$, which are not Dixmier traces and such that $\rho\left(T_{\Delta}\right)>0$, exist by a variant of the argument after (2.45). Correspondingly

$$
\rho\left(P_{a} T_{\Delta}\right)=\frac{\rho\left(T_{\Delta}\right)}{\operatorname{Vol}(M)} \int_{M} a(x) d x, \quad a \in C^{\infty}(M)
$$

provides the integral on a manifold for smooth functions up to a non-zero constant.

The result (4.5) raises the question about whether the class of Dixmier traces $\left(\mathcal{V}_{1}\right)$ is too restrictive in the definition of the noncommutative integral (1.1). If we define

$$
\mathcal{V}_{\text {sing }}^{+}:=\left\{0<\rho \in \mathcal{M}_{1, \infty}^{*} \mid \rho \text { is a singular trace }\right\}
$$

and then define as the 'integral'

$$
\rho\left(a\langle D\rangle^{-n}\right), \quad a \in \mathcal{A}, \quad \rho \in \mathcal{V}_{\text {sing }}^{+}, \quad \rho\left(\langle D\rangle^{-n}\right)>0
$$

we have the same claim to formulas (4.1) and (4.3). Of course, there are a great many formulas involving Dixmier traces besides (4.1) and (4.3), particularly in the development of (local) index theory in noncommutative geometry $[47,16,19,48,49,50,51,52]$. There are geometric consequences of the formulation involving the Dixmier trace and associations to pseudo-differential operators $[17,53,54,55]$, including extensions of Connes' trace theorem $[56,57,53]$. There are 
also many uses of the Dixmier trace in genuine noncommutative examples, for example in the geometry of totally disconnected sets [58, 59, 20].

Are there any of these results for which the submajorisation property of the Dixmier trace is a necessity (recall Dixmier traces correspond to the fully symmetric singular functionals on $m_{1, \infty}$ from Section 2.1)? Several current proofs depend on submajorisation, e.g. residue and heat kernel results are dependent on submajorisation (cf. proofs in [15] and [33]). It is open whether this dependence is a necessity for the results or an artifact of the proofs.

\subsection{Normal extension}

To make things simpler, we dispense with the constants for the moment. Define the noncommutative integral as

$$
\Phi_{\rho}(a)=\rho\left(a\langle D\rangle^{-n}\right), \quad a \in \mathcal{A}, \quad \rho \in \mathcal{V}_{\text {sing }}^{+}, \quad \rho\left(\langle D\rangle^{-n}\right)=1 .
$$

By the discussion in the introduction, the same formula $\Phi_{\rho}$ defines a state of $A$ and $\mathcal{A}^{\prime \prime}$.

Let $M$ be a compact $n$-dimensional Riemannian manifold, $\mathcal{A}=C^{\infty}(M)$ acting by multipliers on $L^{2}(M)$, and $d+d^{*}$ the Hodge-Dirac operator acting (densely) on square integrable sections of the exterior bundle. Then

$$
\left\langle d+d^{*}\right\rangle^{-n}=(1+\Delta)^{-n / 2}=: T_{\Delta}
$$

which we take as acting as a compact operator on $L^{2}(M)$. By $(4.5)$

$$
\Phi_{\rho}(f)=\int_{M} f d \hat{x}, \quad f \in C^{\infty}(M)
$$

where $d \hat{x}$ is the normalised volume form. Recall $\Phi_{\rho}$ is a state on $L^{\infty}(M)$. Hence, by uniform continuity of both the left hand side and right hand side of (4.9)

$$
\Phi_{\rho}(f)=\int_{M} f d \hat{x}, \quad f \in C(M),
$$

which identifies $\left.\Phi_{\rho}\right|_{C(M)}$ with the normalised integral on $C(M)$. Hence $\left.\Phi_{\rho}\right|_{C(M)} \in C(M)^{*}$ has an extension to $L^{\infty}(M)$ given by the normalised integral

$$
\phi(f)=\int_{M} f d \hat{x}, \quad f \in L^{\infty}(M) .
$$

If one considers for the moment a homogeneous compact manifold $M$, by inspection $\Phi_{\rho} \in$ $L^{\infty}(M)^{*}$ is a invariant state, and one might think it is obvious that we must have $\Phi_{\rho}=\phi$ since the volume form provides the unique invariant measure class on $M$. While this last phrase is true, there is no reason a priori why the state $\Phi_{\rho} \in L^{\infty}(M)^{*}$ corresponds to a measure. We know that there can be an infinitude of invariant states on $L^{\infty}(M)$ which all agree with the Lebesgue integral on $C(M)$ [60, Theorem 3.4] (and first Baire class functions ${ }^{6}$ ).

It was not until recently that an elementary argument ${ }^{7}$ was found proving $\Phi_{\rho}=\phi$ for the $n$ torus. An earlier claim [42, p. 297], applied monotone convergence to both sides of (4.9) to extend from $C^{\infty}$-functions to $L^{\infty}$-functions. This method of proof is circular, monotone convergence can be applied to the right hand side, since the integral is a normal linear function on $L^{\infty}(M)$. To apply monotone convergence to the left hand side it must be known $\Phi_{\rho} \in L^{\infty}(M)_{*}$.

In [61] we used a different method than that for torii to show the following result. We remark that [61] concentrated only on Dixmier traces. We state the result for general singular traces here.

\footnotetext{
${ }^{6}$ We are indebted to B. de Pagter for pointing this out and bringing Rudin's paper to our attention. We also thank P. Dodds for additional explanation.

${ }^{7}$ The generalisation of this argument is discussed in our final section, Section 6 .
} 
Theorem 4.3. Let $M$ be a compact $n$-dimensional Riemannian manifold, $\Delta$ the Hodge-Laplacian on $L^{2}(M)$, and $T_{\Delta}$ as above. Then $f T_{\Delta} \in \mathcal{M}_{1, \infty}\left(L^{2}(M)\right)$ if and only if $f \in L^{2}(M)$ and

$$
\Phi_{\rho}(f):=\rho\left(f T_{\Delta}\right)=\int_{M} f d \hat{x}, \quad \forall f \in L^{2}(M)
$$

for any singular trace $0<\rho \in \mathcal{M}_{1, \infty}\left(L^{2}(M)\right)^{*}$ such that $\rho\left(T_{\Delta}\right)=1$.

Proof. We adapt only those parts of [61, Theorem 2.5] concerning (4.10). Define the sequence space

$$
m_{0}=\overline{\ell^{1}} \text { in the norm of } m_{1, \infty}
$$

and the ideal $\mathcal{M}_{0}$ given by $m_{0}=j_{\mathcal{M}_{0}}$. The Riesz seminorm on $\mathcal{M}_{1, \infty}$ is defined by

$$
\|T\|_{0}:=\inf _{Q \in \mathcal{M}_{0}}\|T-Q\|_{1, \infty}
$$

By construction every singular functional $0<\rho \in \mathcal{M}_{1, \infty}^{*}$ vanishes on $\mathcal{M}_{0}$. Hence

$$
|\rho(T)| \leq\|\rho\|\|T\|_{0}, \quad T \in \mathcal{M}_{1, \infty}
$$

The proof in [61] involved, firstly, showing that

$$
\left\|f T_{\Delta}\right\|_{0} \leq C\|f\|_{2}\left\|T_{\Delta}\right\|_{1, \infty}
$$

for a constant $C>0$ (achieved by [61, Corollary 4.5] and [61, Examples 4.6, 4.7]). Hence, from the preceeding two equations, $f T_{\Delta} \in \mathcal{M}_{1, \infty}$ and

$$
\left|\rho\left(f T_{\Delta}\right)\right| \leq C\|\rho\|\|f\|_{2}\left\|T_{\Delta}\right\|_{1, \infty}
$$

for all $f \in C^{\infty}(M)$. Moreover, if $C^{\infty}(M) \ni f_{n} \rightarrow f \in L^{2}(M)$ in the $L^{2}$-norm then $f T_{\Delta}$ is bounded, indeed $f T_{\Delta} \in \mathcal{M}_{1, \infty}$, and

$$
\left|\rho\left(\left(f-f_{n}\right) T_{\Delta}\right)\right| \leq C\|\rho\|\left\|f-f_{n}\right\|_{2}\left\|T_{\Delta}\right\|_{1, \infty} \rightarrow 0 .
$$

The result (4.10) now follows from Connes' trace theorem (specifically, from (4.9)).

As a corollary,

$$
\Phi_{\rho}(f)=\int_{M} f d \hat{x}, \quad f \in L^{\infty}(M)
$$

and we have an example where

$$
\Phi_{\rho} \in L^{\infty}(M)_{*}
$$

(i.e. the noncommutative integral is normal). 


\subsection{Tools for the noncommutative integral}

As mentioned in the introduction, when

$$
\Phi_{\rho}(a)=\rho\left(a\langle D\rangle^{-n}\right), \quad a \in \mathcal{A}^{\prime \prime}, \quad \rho \in \mathcal{V}_{\text {sing }}^{+}, \quad \rho\left(\langle D\rangle^{-n}\right)=1
$$

defines a normal state on the von Neumann algebra $\mathcal{A}^{\prime \prime} \subset \mathcal{L}(H)$, there are Radon-Nikodym theorems, dominated convergence theorems, etc. for this state.

Very little is known about equivalent measure-theoretic results when (4.13) is not known to be normal (and hence the emphasis at present centres on characterising normality in (4.13), see Section 6).

Define the classical weak $\ell^{p}$-spaces (or equivalently the fully symmetric Lorentz sequence spaces $\left.\ell^{p, \infty}\right)$ :

$$
\ell^{p, w}=\left\{x \in c_{0} \mid x^{*}(n)=O\left(n^{-\frac{1}{p}}\right)\right\}, \quad 1 \leq p<\infty .
$$

Denote the corresponding closed fully symmetric ideals of compact operators by $\mathcal{L}^{p, w}$ (i.e. $\ell^{p, w}=$ $\left.j_{\mathcal{L}^{p, w}}\right)$. As is classically known, $\ell^{p, w}$ is the $p$-convexification of $\ell^{1, w}, 1 \leq p<\infty$ [62]. Similarly, from [15] or [38], $\mathcal{L}^{p, w}$ is the $p$-convexification of $\mathcal{L}^{1, w}$. The $p$-convexification $\mathcal{M}_{p, \infty}$ of the ideal $\mathcal{M}_{1, \infty}$, denoted $\mathcal{Z}_{p}$ in [15], is strictly larger that $\mathcal{L}^{p, w}$ (see [15]) .

We do know, from F. Cipriani, D. Guido, and S. Scarlatti [14], and later Guido and T. Isola [20], that Dixmier traces satisfy a Hölder inequality. The result of [20, Theorem 5.1] applies to all singular traces $\rho$ used in (4.13).

Theorem 4.4 ([14, Lemma 1.4], [20, Theorem 5.1], [15, Lemma 6.2(i)]). For $T \in \mathcal{M}_{p, \infty}$ and $V \in \mathcal{M}_{q, \infty}, p, q>1, p^{-1}+q^{-1}=1$, and a singular trace $0<\rho \in \mathcal{M}_{1, \infty}^{*}$, we have $T V \in \mathcal{M}_{1, \infty}$ and the Hölder inequality:

$$
|\rho(T V)| \leq \rho(|T V|) \leq C_{p} \rho\left(|T|^{p}\right)^{\frac{1}{p}} \rho\left(|V|^{q}\right)^{\frac{1}{q}}
$$

where $C_{p}:=1$ if $\rho \in \mathcal{V}_{1}$ and $C_{p}:=1+2 \frac{\sqrt{p-1}}{p}$ otherwise. The inequality also holds for $T \in \mathcal{M}_{1, \infty}$ and $V \in \mathcal{L}(H)$ :

$$
|\rho(T V)| \leq \rho(|T V|) \leq\|V\| \rho(|T|)
$$

(the $p=1, q=\infty$ case).

The Hölder inequality is used to prove that $\rho\left(a\langle D\rangle^{-n}\right)$ is a trace on $\mathcal{A}$ when $(\mathcal{A}, H, D)$ is a spectral triple and $\rho \in \mathcal{V}_{1}[14],[15$, Theorem 6.1]. In [20] it was noted the same proof applies to any singular trace.

Corollary 4.1. Let $(\mathcal{A}, H, D)$ be a spectral triple such that $\langle D\rangle^{-n} \in \mathcal{M}_{1, \infty}$. Then (4.7) (resp. (4.13)) defines a finite positive trace (resp. trace state) on $\mathcal{A}$.

\footnotetext{
${ }^{8}$ Note that the $(p, \infty)$-summable condition for K-cycles (spectral triples) is sometimes stated as $\langle D\rangle^{-1} \in \mathcal{L}^{p, w}$, $1 \leq p<\infty$ [2, p. 546], [3, p. 159] (equivalent to $\langle D\rangle^{-p} \in \mathcal{L}^{1, w}$ ) and sometimes as $\langle D\rangle^{-1} \in \mathcal{M}_{1, \infty}(p=1)$ and $\langle D\rangle^{-1} \in \mathcal{L}^{p, w}(p>1)$ [2, IV.2] (not equivalent to $\langle D\rangle^{-p} \in \mathcal{L}^{1, w}$ or $\langle D\rangle^{-p} \in \mathcal{M}_{1, \infty}$ ). To distinguish the strictly smaller ideal $\mathcal{L}^{1, w}$ from the usually quoted domain of the Dixmier trace (the ideal $\mathcal{M}_{1, \infty}$ ) we have avoided the notation $\mathcal{L}^{1, \infty}$ for $\mathcal{M}_{1, \infty}$.
} 


\section{$5 \quad$ Zeta functions and heat kernels}

Zeta functions and heat kernel asymptotics are alternative ways to measure 'the log divergence of the trace'.

The Wodzicki residue Res on classical pseudo-differential operators of order $-n$ on a $n$ dimensional compact Riemannian manifold $M$ derives its name as the noncommutative residue from the zeta function formulation of the residue for positive elliptic pseudo-differential operators $[43,63]$.

Explicitely, if $0<Q \in \mathrm{Op}_{\mathrm{cl}}^{d}(M), d>0$, is elliptic, then $Q^{-s}, s>n / d$, is trace class $[64,44,46]$. It is known the function

$$
\zeta_{Q}(s):=\operatorname{Tr}\left(Q^{-s}\right), \quad s>n / d,
$$

called the zeta function and initially introduced for the Laplacian [65] has a meromorphic continuation with simple pole at $s=n / d[64,66]$ and from [43]

$$
\operatorname{res}_{s=n / d} \zeta_{Q}(s)=\lim _{s \rightarrow(n / d)^{+}}(s-n / d) \operatorname{Tr}\left(Q^{-s}\right)=-\frac{1}{d} \operatorname{Res}\left(Q^{-n / d}\right) .
$$

The Wodzicki residue has a similar identification involving the heat kernel operator $e^{-t Q}, t>0$. The heat kernel operator is so named since the kernel $K(t, x, y) \in C^{\infty}((0, \infty) \times M \times M)$ associated to the trace-class family $e^{-t \Delta}, t>0, \Delta$ the Hodge Laplacian,

$$
\left(e^{-t \Delta} h\right)(x)=\int_{M} K(t, x, y) h(y) d y, \quad h \in L^{2}(M)
$$

is a solution to the (local) heat equation [65]

$$
\left(\partial_{t}-\Delta_{x}\right) K(t, x, y)=0, \quad \lim _{t \rightarrow 0^{+}} K(t, x, y)=\delta_{y}(x),
$$

where $\delta$ is the Dirac delta function and the limit is in the weak sense. The zeta function and the trace of the heat kernel operator are related by the Mellin transform

$$
\Gamma(s) \zeta_{Q}(s)=\int_{0}^{\infty} t^{s-1} \operatorname{Tr}\left(e^{-t Q}\right) d t .
$$

Using the asymptotic expansion of the heat kernel operator $[65,67,68]$ the Wodzicki residue is associated to the heat kernel by

$$
\lim _{t \rightarrow 0^{+}} t^{n / d} \operatorname{Tr}\left(e^{-t Q}\right)=\Gamma\left(\frac{n}{d}\right) \operatorname{res}_{s=n / d} \zeta_{Q}(s)=-\frac{1}{d} \Gamma\left(\frac{n}{d}\right) \operatorname{Res}\left(Q^{-n / d}\right) .
$$

From Connes' trace theorem (Theorem 4.1 and 4.2) the Dixmier trace of $\langle D\rangle^{-n}$ has claim to be the noncommutative version of the Wodzicki residue $\operatorname{Res}\left(Q^{-n}\right), 0<Q \in \mathrm{Op}_{\mathrm{cl}}^{1}(M)$. Do residue and heat kernel formulas similar to (5.1) and (5.2) hold for Dixmier traces? Sections 5.1 and 5.2 list what is known.

We detail only the latest known identifications between Dixmier traces and residues and heat kernels. For applications of the residue and heat kernel to index formulations in NCG see $[47,19,49,50,52]$.

\subsection{Residues of zeta functions}

A. Connes introduced the association between a generalised zeta function,

$$
\zeta_{T}(s):=\operatorname{Tr}\left(T^{s}\right)=\sum_{n=1}^{\infty} \mu_{n}(T)^{s}, \quad 0<T \in \mathcal{M}_{1, \infty}
$$


and the calculation of a Dixmier trace with the result that

$$
\lim _{s \rightarrow 1^{+}}(s-1) \zeta_{T}(s)=\lim _{N \rightarrow \infty} \frac{1}{\log (1+N)} \sum_{n=1}^{N} \mu_{n}(T)
$$

if either limit exists [2, p. 306]. Meromorphicity of the formula (5.3) is discussed in $[47,69,66]$. Generalisations of (5.4) appeared in [25] and later [15].

In [70] we translated the results [15, Theorem 4.11] and [25, Theorem 3.8] to $\ell^{\infty}$, see Theorem 5.1 and Corollary 5.1 below. Recall $\mathcal{T}^{+}=\mathcal{K}_{1}^{+}=\mathcal{K}_{2}^{+}(3.5)$, so

$$
\operatorname{Tr}_{\omega}(T)=\lim _{s \rightarrow 1^{+}}(s-1) \zeta_{T}(s)
$$

calculates the Dixmier or Connes-Dixmier trace of any (Dixmier or Connes-Dixmier) measurable positive operator $0<T \in \mathcal{M}_{1, \infty}$ as the residue at $s=1$ of the zeta function $\zeta_{T}$. What about general $0<T \in \mathcal{M}_{1, \infty}$ ?

The zeta function of a positive compact operator $T$ given by (5.3) relies on the assumption that there exists some $s_{0}$ for which $T^{s}$ is trace class $s>s_{0}$ (equally $T \in \mathcal{L}^{s}$ for $s>s_{0}$ ). For convenience we assume $s_{0}=1$. The space of compact operators for which the zeta function exists and $(s-1) \zeta_{T}(s) \in L^{\infty}((1,2])$ was studied in [15]. Define the norm

$$
\|T\|_{\mathcal{Z}_{1}}:=\limsup _{s \rightarrow 1^{+}}(s-1)\|T\|_{s}
$$

where $\|T\|_{s}=\operatorname{Tr}\left(|T|^{s}\right)^{\frac{1}{s}}$. Then $[15$, Theorem 4.5] identified that

$$
\mathcal{Z}_{1}=\left\{T \in \mathcal{L}^{\infty} \mid\|T\|_{\mathcal{Z}_{1}}<\infty\right\} \equiv \mathcal{M}_{1, \infty}
$$

and that

$$
e^{-1}\|T\|_{0} \leq\|T\|_{\mathcal{Z}_{1}} \leq\|T\|_{1, \infty}
$$

where $\|\cdot\|_{0}$ is the Riesz seminorm (4.12).

Hence, $(s-1) \zeta_{|T|}(s) \in L^{\infty}((1,2])$ if and only if $T \in \mathcal{M}_{1, \infty}$, which we rewrite as (defining the following function to be 0 for $r \in[0,1)$ )

$$
\frac{1}{r} \zeta_{|T|}\left(1+\frac{1}{r}\right) \in L^{\infty}([0, \infty)) \Leftrightarrow T \in \mathcal{M}_{1, \infty}
$$

This is known, from [70], to be equivalent to

$$
\frac{1}{k} \zeta_{|T|}\left(1+\frac{1}{k}\right) \in \ell^{\infty} \Leftrightarrow T \in \mathcal{M}_{1, \infty}
$$

We can obtain positive unitarily invariant singular functionals on $\mathcal{M}_{1, \infty}$, that will equate to (5.5) when $T$ is a Tauberian operator, by applying a generalised limit $\xi \in S_{\infty}\left(\ell^{\infty}\right)$ (resp. $\phi \in$ $\left.S_{\infty}([0, \infty))\right)$ to the sequence (5.7) (resp. function (5.6)). Exactly which generalised limits produce linear functionals (and hence singular traces) is an open question. We know that choosing $\xi \in B L \cap D L$ (resp. $\phi \in B L[0, \infty) \cap D L[0, \infty)$ ) results in a Dixmier trace.

We summarise the results of [70], based on [15, Theorem 4.11], see [25] and [19] for additional information. Define the averaging sequence $E: L^{\infty}([0, \infty)) \rightarrow \ell^{\infty}$ by

$$
E_{k}(f):=\int_{k-1}^{k} f(t) d t, \quad f \in L^{\infty}([0, \infty)) .
$$


Define the map $L^{-1}: L^{\infty}([1, \infty)) \rightarrow L^{\infty}([0, \infty))$ by

$$
L^{-1}(g)(t)=g\left(e^{t}\right), \quad g \in L^{\infty}([1, \infty)) .
$$

Define the floor map $p: \ell^{\infty} \rightarrow L^{\infty}([1, \infty))$ by

$$
p\left(\left\{a_{k}\right\}_{k=1}^{\infty}\right)(t):=\sum_{k=1}^{\infty} a_{k} \chi_{[k, k+1)}(t), \quad\left\{a_{k}\right\}_{k=1}^{\infty} \in \ell^{\infty} .
$$

Define, finally, the mapping $\mathcal{L}:\left(\ell^{\infty}\right)^{*} \rightarrow\left(\ell^{\infty}\right)^{*}$ by

$$
\mathcal{L}(\omega):=\omega \circ E \circ L^{-1} \circ p, \quad \omega \in\left(\ell^{\infty}\right)^{*} .
$$

In the following statements we use the notation established in (2.8)-(2.12) and (2.21)-(2.29).

Theorem 5.1 ([70, Theorems 3.1, 3.3]). Let $P^{*}=P=P^{2} \in \mathcal{L}(H)$ be a projection and $0<T \in \mathcal{M}_{1, \infty}$.

Then, for any $\phi \in B L[0, \infty) \cap D L[0, \infty)$,

$$
\operatorname{tr}_{L(\phi)}(P T P)=\phi\left(\frac{1}{r} \operatorname{Tr}\left(P T^{1+\frac{1}{r}} P\right)\right) .
$$

Similarly, for any $\xi \in B L \cap D L, \mathcal{L}(\xi) \in D_{2}$ and

$$
\operatorname{Tr}_{\mathcal{L}(\xi)}(P T P)=\xi\left(\frac{1}{k} \operatorname{Tr}\left(P T^{1+\frac{1}{k}} P\right)\right)
$$

Moreover, $\lim _{s \rightarrow 1^{+}}(s-1) \operatorname{Tr}\left(P T^{s} P\right)$ exists iff PTP is Tauberian and in either case

$$
\operatorname{tr}_{v}(P T P)=\operatorname{Tr}_{\omega}(P T P)=\lim _{s \rightarrow 1^{+}}(s-1) \operatorname{Tr}\left(P T^{s} P\right)
$$

for all $v \in D L[1, \infty), \omega \in D_{2}$.

Corollary 5.1 ([70, Corollaries 3.2, 3.4]). Let $A \in \mathcal{L}(H)$ and $0<T \in \mathcal{M}_{1, \infty}$. Then, for any $\phi \in B L[0, \infty) \cap D L[0, \infty)$,

$$
\operatorname{tr}_{L(\phi)}(A T)=\phi\left(\frac{1}{r} \operatorname{Tr}\left(A T^{1+\frac{1}{r}}\right)\right) .
$$

Similarly, for any $\xi \in B L \cap D L$,

$$
\operatorname{Tr}_{\mathcal{L}(\xi)}(A T)=\xi\left(\frac{1}{k} \operatorname{Tr}\left(A T^{1+\frac{1}{k}}\right)\right)
$$

Moreover, if PTP is Tauberian for all projections $P$ in the von Neumann algebra generated by $A$ and $A^{*}$,

$$
\operatorname{tr}_{v}(A T)=\operatorname{Tr}_{\omega}(A T)=\lim _{s \rightarrow 1^{+}}(s-1) \operatorname{Tr}\left(A T^{s}\right)
$$

for all $v \in D L[1, \infty), \omega \in D_{2}$.

For the situation $s_{0}=p$, see [15]. 


\section{$5.2 \quad$ Heat kernel asymptotics}

We follow the exposition of [32], following [25] and [15]. From (2.7) and (2.19)

$$
L(C)(f)(t)=\frac{1}{\log t} \int_{1}^{t} f(s) \frac{d s}{s}, \quad f \in L^{\infty}([1, \infty)) .
$$

It was noted in [15, Lemma 5.1] that the function

$$
g_{T, \alpha}(t)=\frac{1}{t} \operatorname{Tr}\left(e^{-(t T)^{-\alpha}}\right), \quad \alpha>0
$$

belongs to $L^{\infty}([1, \infty))$ only for $0<T \in \mathcal{L}^{1, w}$, where $\mathcal{L}^{1, w}$ is the symmetric subspace from $(3.13)$ or (4.14). In general we have only

$$
L(C)\left(g_{T, \alpha}\right) \in L^{\infty}([1, \infty)), \quad 0<T \in \mathcal{M}_{1, \infty} .
$$

For this reason heat kernel results are likely to be restricted only to Connes-Dixmier traces $\left(\mathcal{V}_{2}\right)$ or Cesàro invariant Dixmier traces $\left(\mathcal{V}_{3}\right)$ outside of Tauberian operators in $\mathcal{L}^{1, w}$. From [32] and [33]:

Theorem 5.2 ([32, Theorems 3-6] and [33, Theorem 33]). Let $\omega \in C D L[1, \infty)$ and $0<T \in \mathcal{M}_{1, \infty}$ have trivial kernel. Then

$$
\operatorname{tr}_{\omega}(T)=\frac{1}{\Gamma\left(\frac{1}{\alpha}+1\right)} \omega \circ L(C)\left(\frac{1}{t} \operatorname{Tr}\left(e^{-(t T)^{-\alpha}}\right)\right), \quad \alpha>0 .
$$

Let $\omega \in C D L[1, \infty)$ and $0<T \in \mathcal{L}^{1, w}$ have trivial kernel. Then

$$
\operatorname{tr}_{\omega}(T)=\frac{1}{\Gamma\left(\frac{1}{\alpha}+1\right)} \omega\left(\frac{1}{t} \operatorname{Tr}\left(e^{-(t T)^{-\alpha}}\right)\right), \quad \alpha>0 .
$$

Let $\omega \in D(C)[1, \infty)$ and $0<T \in \mathcal{M}_{1, \infty}$ have trivial kernel. Then

$$
\operatorname{tr}_{\omega}(T)=\frac{1}{\Gamma\left(\frac{1}{\alpha}+1\right)} \omega\left(\frac{1}{t} \operatorname{Tr}\left(e^{-(t T)^{-\alpha}}\right)\right), \quad \alpha>0
$$

Finally, if $0<T \in \mathcal{L}^{1, w}$ is Tauberian with trivial kernel, then

$$
\operatorname{tr}_{\omega}(T)=\frac{1}{\Gamma\left(\frac{1}{\alpha}+1\right)} \lim _{t \rightarrow \infty} \frac{1}{t} \operatorname{Tr}\left(e^{-(t T)^{-\alpha}}\right)=\frac{1}{\Gamma\left(\frac{1}{\alpha}+1\right)} \lim _{t \rightarrow 0^{+}} t^{\frac{1}{\alpha}} \operatorname{Tr}\left(e^{-t T^{-\alpha}}\right), \quad \alpha>0
$$

for all $\omega \in D L[1, \infty)$.

For example, if $\langle D\rangle^{-n}$ is known to be Tauberian and belongs to $\mathcal{L}^{1, w}$ then

$$
\operatorname{tr}_{\omega}\left(\langle D\rangle^{-n}\right)=\frac{1}{\Gamma\left(\frac{n}{2}+1\right)} \lim _{t \rightarrow 0^{+}} t^{\frac{n}{2}} \operatorname{Tr}\left(e^{-t D^{2}}\right) .
$$

From Weyl's formula for the eigenvalues of the Hodge Laplacian on a $n$-dimensional compact Riemannian manifold (and also using comments from [15, p. 278]), we have that

$$
0<\left(1+Q^{2}\right)^{-n / 2 d} \in \mathcal{L}^{1, w}
$$

and is Tauberian for any positive elliptic operator in $Q \in \mathrm{Op}_{\mathrm{cl}}^{d}(M)$. This fact, combined with the above equations, reconstructs (5.2).

For heat kernel formulas involving $\operatorname{tr}_{\omega}(A T), A \in \mathcal{L}(H), 0<T \in \mathcal{M}_{1, \infty}$, see [25] and [15]. 


\section{Characterising the noncommutative integral}

The functional

$$
\Phi_{\mathrm{f}, T}(a):=\mathrm{f}(a T), \quad a \in \mathcal{L}(H),
$$

where $\mathrm{f}$ is a trace on a two-sided ideal of compact operators $\mathcal{J}$ and $0<T \in \mathcal{J}$, is the general format of noncommutative integration introduced by Connes. We are concerned in this section with the characterisation of linear functionals on $\mathcal{L}(H)$ constructed by (6.1).

The functional $\Phi_{\mathrm{f}, T}$ is normal (contained in $\mathcal{L}(H)_{*}$ ) if and only if $\Phi_{\mathrm{f}, T}(a)=\operatorname{Tr}(a T), \forall a \in$ $\mathcal{L}(H)$, for a positive trace class 'density' $T$. From the definition of the canonical trace $\operatorname{Tr}$ :

$$
\operatorname{Tr}(a T)=\sum_{m=1}^{\infty} \lambda_{m}\left\langle h_{m}, a h_{m}\right\rangle,
$$

where $\left\{h_{m}\right\}_{m=1}^{\infty}$ is an orthonormal basis of eigenvectors for the positive compact operator $T$ with eigenvalues $0<\left\{\lambda_{m}\right\}_{m=1}^{\infty} \in \ell^{1}$. Define the normal linear functional on $\ell^{\infty}, \sigma_{T} \in\left(\ell^{\infty}\right)_{*} \cong \ell^{1}$, by

$$
\sigma_{T}\left(\left\{a_{m}\right\}_{m=1}^{\infty}\right):=\sum_{m=1}^{\infty} \lambda_{m} a_{m} .
$$

Then

$$
\operatorname{Tr}(a T)=\sigma_{T}\left(\left\{\left\langle h_{m}, a h_{m}\right\rangle\right\}_{m=1}^{\infty}\right), \quad \forall a \in \mathcal{L}(H) .
$$

Conversely,

$$
\sigma\left(\left\{\left\langle h_{m}, a h_{m}\right\rangle\right\}_{m=1}^{\infty}\right), \quad 0<\sigma \in\left(\ell^{\infty}\right)_{*}, \quad a \in \mathcal{L}(H)
$$

defines a positive normal linear functional on $\mathcal{L}(H)$ for any choice of orthonormal basis $\left\{h_{m}\right\}_{m=1}^{\infty}$ of $H$. If $\mathbf{e}_{m}$ is the sequence with 1 in the $m^{\text {th }}$ place and 0 otherwise, set $T_{\sigma}$ to be the operator with eigenvalues $0<\left\{\sigma\left(\mathbf{e}_{m}\right)\right\}_{m=1}^{\infty} \in \ell_{1}$ associated to the basis $\left\{h_{m}\right\}_{m=1}^{\infty}$. By this construction

$$
\operatorname{Tr}\left(a T_{\sigma}\right)=\sigma\left(\left\{\left\langle h_{m}, a h_{m}\right\rangle\right\}_{m=1}^{\infty}\right), \quad \forall a \in \mathcal{L}(H) .
$$

By (6.2) and (6.3) the positive normal linear functionals

$$
\Phi_{\operatorname{Tr}, T}(a)=\operatorname{Tr}(a T), \quad 0<T \in \mathcal{L}^{1}
$$

are exactly characterised by

$$
0<\sigma \in\left(\ell^{\infty}\right)_{*} \text { applied to the sequence of "expectation values" }\left\{\left\langle h_{m}, a h_{m}\right\rangle\right\}_{m=1}^{\infty}
$$

for some orthonormal basis $\left\{h_{m}\right\}_{m=1}^{\infty}$. We now know that a similar characterisation exists for any functional $\Phi_{\mathrm{f}, T}$ as defined in (6.1).

\subsection{Characterisation and singular traces}

Let $\mathcal{J}$ be a (two sided) ideal contained in the compact operators $\mathcal{L}^{\infty}$ of the separable complex Hilbert space $H$. By a trace on $\mathcal{J}$ we mean a linear functional $\mathrm{f}: \mathcal{J} \rightarrow \mathbb{C}$ such that $\mathrm{f}([a, T])=0$ for all $a \in \mathcal{L}(H), T \in \mathcal{J}$, i.e. f vanishes on the commutator subspace Com $\mathcal{J}$ of $\mathcal{J}$. Note it is not assumed that $\mathrm{f}$ is positive or continuous.

The characterisation for (6.1) in Theorem 6.1 below was first shown for $\mathrm{f} \in \mathcal{V}_{P D L[1, \infty)}$, $0<T \in \mathcal{M}_{1, \infty}$, in [70, Theorem 3.6] using residues (Corollary 5.1). The result below (to appear) is due to collaboration with N. Kalton and uses results on the commutator subspace and sums of commutators [71, 72]. 
Theorem 6.1. Let $0<T \in \mathcal{J}$ and $\mathrm{f}$ be a trace on $\mathcal{J}$. Then there exists an orthonormal basis $\left\{h_{m}\right\}_{m=1}^{\infty}$ of $H$ (consisting of eigenvectors for $T$ ) and a linear functional $\sigma: \ell^{\infty} \rightarrow \mathbb{C}$ such that

$$
\mathrm{f}(a T)=\sigma\left(\left\{\left\langle h_{m}, a h_{m}\right\rangle\right\}_{m=1}^{\infty}\right)
$$

for all $a \in \mathcal{L}(H)$.

Straightforward corollaries show, when $\mathrm{f}>0$, that $\mathrm{f}$ has the property

$$
|\mathrm{f}(a T)| \leq C\|a\| \text { some } C>0,
$$

if and only if $0<\sigma \in\left(\ell^{\infty}\right)^{*}$. The basis $\left\{h_{m}\right\}_{m=1}^{\infty}$ in the theorem is any basis of eigenvectors of $T$ rearranged so that the sequence of eigenvalues $\lambda_{m}$ associated to $h_{m}$ is a decreasing sequence.

A singular trace is a trace on a two sided ideal $\mathcal{J}$, containing the finite rank operators $F$, that vanishes on $F \subset \mathcal{J}$. The theorem has the following corollary.

Corollary 6.1. Let $0<T \in \mathcal{J}$ and $\mathrm{f}$ be a positive singular trace on $\mathcal{J}$. Then there exists an orthonormal basis $\left\{h_{m}\right\}_{m=1}^{\infty}$ of $H$ (consisting of eigenvectors for $T$ ) and a singular state (generalised limit) $L: \ell^{\infty} \rightarrow \mathbb{C}$ such that

$$
\mathrm{f}(a T)=\mathrm{f}(T) L\left(\left\{\left\langle h_{m}, a h_{m}\right\rangle\right\}_{m=1}^{\infty}\right)
$$

for all $a \in \mathcal{L}(H)$.

In particular, for the noncommutative integral from (4.13),

$$
\Phi_{\rho}(a)=\rho\left(a\langle D\rangle^{-n}\right), \quad a \in \mathcal{A}^{\prime \prime}, \quad \rho \in \mathcal{V}_{\text {sing }}^{+}, \quad \rho\left(\langle D\rangle^{-n}\right)=1
$$

the corollary implies that there exists a basis of eigenvectors $\left\{h_{m}\right\}_{m=1}^{\infty}$ of $D$ such that

$$
\rho\left(a\langle D\rangle^{-n}\right)=L_{\rho}\left(\left\{\left\langle h_{m}, a h_{m}\right\rangle\right\}_{m=1}^{\infty}\right)
$$

where $L_{\rho}$ is a generalised limit.

Example 6.1. Let $\mathbb{T}^{n}$ be the $n$-torus, $\mathbb{R}^{n} / \mathbb{Z}^{n}$, with Laplacian $\Delta$. Let $\mathcal{J}$ be any two-sided ideal with trace $\mathrm{f}$ and $g$ be a bounded Borel function $g$ such that $g(\Delta) \in \mathcal{J}$. Let $f \in L^{\infty}\left(\mathbb{T}^{n}\right)$ and denote by

$$
f g(\Delta)
$$

the action of $f$ on $L^{2}\left(\mathbb{T}^{n}\right)$ by pointwise multiplication coupled with the compact operator $g(\Delta)$, c.f. $[11, \S 4]$. Then, by Theorem 6.1 ,

$$
\mathrm{f}(f g(\Delta))=\sigma\left(\left\langle e^{i \mathbf{m} \cdot x}, f e^{i \mathbf{m} \cdot x}\right\rangle\right)
$$

where $\mathbf{m} \in \mathbb{Z}^{n}$ (ordered by Cantor enumeration) and $\sigma$ is a linear functional on $\ell^{\infty}$. As

$$
\left\langle e^{i \mathbf{m} \cdot x}, f e^{i \mathbf{m} \cdot x}\right\rangle=\int_{\mathbb{T}^{n}} e^{-i \mathbf{m} \cdot x} f(x) e^{i \mathbf{m} \cdot x} d x=\int_{\mathbb{T}^{n}} f(x) d x
$$

and

$$
\sigma(\mathbf{1})=\mathrm{f}(g(\Delta))
$$

we obtain

$$
\mathrm{f}(f g(\Delta))=\mathrm{f}(g(\Delta)) \int_{\mathbb{T}^{n}} f(x) d x .
$$


Therefore, for any ideal $\mathcal{J}$ with a non-trivial trace $\mathrm{f}$, by choosing the appropriate bounded Borel function $g$, the expression

$$
\mathrm{f}(\cdot g(\Delta))
$$

may serve as the integral on the $n$-torus (up to a constant). Note the identification (6.6) holds for all $f \in L^{\infty}\left(\mathbb{T}^{n}\right)$.

Can the same statement as for the $n$-torus in the example above be made for the Lebesgue integral of a compact Riemannian manifold $M$, considering non-trivial positive singular traces instead of arbitrary traces?

We certainly know, from Corollary 6.1 and Theorem 4.3, that if the sequence

$$
\left\{\left\langle h_{m}, f h_{m}\right\rangle\right\}_{m=1}^{\infty} \in c, \quad \forall f \in C^{\infty}(M)
$$

for a basis of eigenvectors $\left\{h_{m}\right\}_{m=1}^{\infty}$ of the Hodge-Laplacian $\Delta$ (ordered so that the eigenvalues associated to the eigenvectors are decreasing), then

$$
\mathrm{f}(f g(\Delta))=\mathrm{f}(g(\Delta)) \int_{M} f d x, \quad \forall f \in C^{\infty}(M)
$$

for any positive non-trivial singular trace $\mathrm{f}$ on an ideal $\mathcal{J}$ and with an appropriate choice of bounded Borel function $g$ (so that $g(\Delta) \in \mathcal{J})$.

The property (6.7) is a restricted form of a property for compact Riemannian manifolds known as Quantum Unique Ergodicity (QUE) [73, 74]. It is not well known which manifolds have the QUE property.

The same result as for the $n$-torus applies to anything 'flat'. The next example involving the non-commutative torus first appeared (for $\mathrm{f} \in \mathcal{V}_{P D L[1, \infty)}$ ) in [70].

Example 6.2. Consider two unitaries $u, v$ such that $u v=\lambda v u$, for $\lambda:=e^{2 \pi i \theta} \in \mathbb{S}$ (the unit circle). Denote by $F_{\theta}(u, v)$ the *-algebra of linear combinations $\sum_{(m, n) \in J} a_{m, n} u^{m} v^{n}$, $J \subset \mathbb{Z}^{2}$ is a finite set, with product $a b=\sum_{r, s}\left(\sum_{m, n} a_{r-m, n} \lambda^{m n} b_{m, s-n}\right) u^{r} v^{s}$ and involution $a^{*}=\sum_{r, s}\left(\lambda^{r s} \bar{a}_{-r,-s}\right) u^{r} v^{s}, a, b \in F_{\theta}(u, v)$. The assignment $\tau_{0}(a)=a_{0,0}$ is a faithful trace on $F_{\theta}(u, v)$. Let $\left(H_{\theta}, \pi_{\theta}\right)$ denote the cyclic representation associated to $\tau_{0}$. The closure, $C_{\theta}(u, v)$, of $\pi_{\theta}\left(F_{\theta}(u, v)\right)$ in the operator norm is called a rotation $C^{*}$-algebra, [75], or the noncommutative torus $(\lambda \neq 1)[2,3,42]$. Canonically, finite linear combinations of $u^{m} v^{n} \hookrightarrow H_{\theta}$ are dense in $H_{\theta}$. Define $\Delta_{\theta}\left(u^{m} v^{n}\right)=\left(m^{2}+n^{2}\right) u^{m} v^{n}$. It can be shown that the 'noncommutative Laplacian' $\Delta_{\theta}$ has a unique positive extension (also denoted $\left.\Delta_{\theta}\right) \Delta_{\theta}: \operatorname{Dom}\left(\Delta_{\theta}\right) \rightarrow H_{\theta}$ with compact resolvent, see the previous citations. The eigenvectors $h_{m, n}=u^{m} v^{n} \in H_{\theta}$ form a complete orthonormal system. Note that

$$
\left\langle h_{m, n}, \pi_{\theta}(a) h_{m, n}\right\rangle=: \tau_{0}\left(\left(u^{m} v^{n}\right)^{*} a u^{m} v^{n}\right)=\tau_{0}(a), \quad \forall a \in F_{\theta}(u, v)
$$

for any $(m, n) \in \mathbb{Z}^{2}$. Using the Cantor enumeration of $\mathbb{Z}^{2}$, from Theorem 6.1 we obtain

$$
\mathrm{f}\left(a g\left(\Delta_{\theta}\right)\right)=\mathrm{f}\left(g\left(\Delta_{\theta}\right)\right) \tau_{0}(a), \quad \forall a \in F_{\theta}(u, v)
$$

for any ideal $\mathcal{J}$ with a non-trivial trace $\mathrm{f}$, by choosing the appropriate bounded Borel function $g$ such that $g\left(\Delta_{\theta}\right) \in \mathcal{J}$. In fact, $\left\langle h_{m, n}, b h_{m, n}\right\rangle=\tau(b)$ for any $b$ in the weak closure $C_{\theta}(u, v)^{\prime \prime}$ of $\pi_{\theta}\left(F_{\theta}(u, v)\right)$ and where $\tau$ denotes the normal extension of $\tau_{0}$. Hence

$$
\mathrm{f}\left(a g\left(\Delta_{\theta}\right)\right)=\mathrm{f}\left(g\left(\Delta_{\theta}\right)\right) \tau(a), \quad \forall a \in C_{\theta}(u, v)^{\prime \prime} .
$$




\subsection{A dominated convergence theorem for the noncommutative integral}

The following dominated convergence theorem appeared in [70] as a statement for Dixmier traces. We provide the general statement here.

Let $\mathcal{N}$ be a weakly closed $*$-subalgebra of $B(H)$. We recall that $\mathcal{N}_{*}$ denotes the predual of $\mathcal{N}$, or the set of all normal linear functionals on $\mathcal{N}$.

We say a positive compact operator $T$ is $(\mathcal{N}, h)$-dominated if, for some orthonormal basis $\left\{h_{m}\right\}_{m=1}^{\infty}$ of eigenvectors of $T$, there exists $h \in H$ such that $\left\|P h_{m}\right\| \leq\|P h\|$ for all projections $P \in \mathcal{N}$.

Theorem 6.2. Let $0<T \in \mathcal{J}$ and $\mathrm{f}$ be a positive singular trace on $\mathcal{J}$. If $T$ is $(\mathcal{N}, h)$-dominated, then $\Phi_{\mathrm{f}, T}=f(\cdot T) \in \mathcal{N}_{*}$.

Proof. Without loss $\left\{h_{m}\right\}_{m=1}^{\infty}$ can be rearranged so that the eigenvalues $\lambda_{m}$ associated to $h_{m}$ form a decreasing sequence. By hypothesis $\left\langle h_{m}, P h_{m}\right\rangle \leq\langle h, P h\rangle$ for all projections $P \in \mathcal{N}$. Then $\left\langle h_{m}, a h_{m}\right\rangle \leq\langle h, a h\rangle, 0<a \in \mathcal{N}$, as $a$ is a uniform limit of finite linear positive spans of projections [10, p. 23]. For any generalised limit $L$ and $0<a \in \mathcal{N}$,

$$
L\left(\left\langle h_{m}, a h_{m}\right\rangle\right) \leq \limsup _{m \rightarrow \infty}\left\langle h_{m}, a h_{m}\right\rangle \leq\langle h, a h\rangle .
$$

Let $\left\{a_{\alpha}\right\}$ be a net of monotonically increasing positive elements of $\mathcal{N}$ with upper bound. It follows that $\left\{a_{\alpha}\right\}$ converges strongly to a l.u.b. $a \in \mathcal{N}\left[10\right.$, p. 22]. From $(6.8) L\left(\left\langle h_{m},\left(a-a_{\alpha}\right) h_{m}\right\rangle\right) \leq$ $\left\langle h,\left(a-a_{\alpha}\right) h\right\rangle$. Since $\left\langle h,\left(a-a_{\alpha}\right) h\right\rangle \stackrel{\alpha}{\rightarrow} 0, L\left(\left\langle h_{m}, a h_{m}\right\rangle\right)=\sup _{\alpha} L\left(\left\langle h_{m}, a_{\alpha} h_{m}\right\rangle\right)$. From Corollary 6.1 $\Phi_{\mathrm{f}, T}(a)=\sup _{\alpha} \Phi_{\mathrm{f}, T}\left(a_{\alpha}\right)$ and $\Phi_{\mathrm{f}, T}$ is normal on $\mathcal{N}[10, \S 3.6 .1]$.

We close with an example of $(\mathcal{N}, h)$-dominated compact operators.

Let $H=L^{2}(X, \mu)$ for a $\sigma$-finite measure space $(X, \mu)$ and set $\mathcal{N}$ as multiplication operators of $L^{\infty}$-functions. Then $0<T \in \mathcal{L}^{\infty}\left(L^{2}(X, \mu)\right)$ being $(\mathcal{N}, h)$-dominated is the same as $\int_{J}\left|h_{m}(x)\right|^{2} d \mu(x) \leq \int_{J}|h(x)|^{2} d \mu(x)$ for all measurable sets $J \subset X$, which is equivalent to the statement $\left|h_{m}\right|^{2}$ are dominated by some $|h|^{2} \in L^{1}(X, \mu) \mu$-a.e., where $\left\{h_{m}\right\}_{m=1}^{\infty}$ is any basis of eigenvectors of $T$. Hence any positive compact operator $0<T \in \mathcal{L}^{\infty}\left(L^{2}(X, \mu)\right)$ is $(\mathcal{N}, \sqrt{g})$ dominated if it has a basis of eigenvectors whose modulus squared are dominated by some positive integrable function $g \in L^{1}(X, \mu)$. For instance, the negative powers of the Laplacian on the $n$-torus are dominated by the constant function $1 \in L^{1}\left(\mathbb{T}^{n}\right)$.

\section{Summary}

We summarise the main points of the review and list the open questions raised.

\subsection{Summary}

There are three main non-identical classes of Dixmier traces. They are, in descending order of inclusion: the (original) Dixmier traces $\left(\mathcal{V}_{1}\right)$; the Connes-Dixmier traces $\left(\mathcal{V}_{2}\right)$; and the Cesàro invariant Dixmier traces $\left(\mathcal{V}_{3}\right)$.

The notion of measurable operator with respect to each of these classes are non-identical. The notion of Tauberian, (3.2), is the strongest notion with ascending order of inclusion

$$
\mathcal{T} \subsetneq \mathcal{K}_{1} \subsetneq \mathcal{K}_{2} \subsetneq \mathcal{K}_{3}
$$

where

$$
\mathcal{K}_{i}=\left\{T \in \mathcal{M}_{1, \infty} \mid \mathrm{f}(T)=\text { const } \forall \mathrm{f} \in \mathcal{V}_{i}\right\}, \quad i=1,2,3 .
$$


None of these sets is an ideal of compact operators. The notion of measurable operator using Dixmier traces does not achieve $\mathcal{T}=\mathcal{K}_{1}$, however $\mathcal{T}^{+}=\mathcal{K}_{1}^{+}$. This has the consequence that the operators $T \in \mathcal{K}_{1}$ do not decompose into unique measurable components. In short, $\mathcal{K}_{1}$ does not have the property $T \in \mathcal{K}_{1} \Rightarrow|T| \in \mathcal{K}_{1}$.

Connes' trace theorem (Theorem 4.1) is valid for any $\rho \in \mathcal{V}_{\text {sing }}^{+},(4.6)$. In particular, if $M$ is an $n$-dimensional compact Riemannian manifold with Hodge Laplacian $\Delta$ we have

$$
\rho(P)=c_{\rho} \operatorname{Res}(P) \quad \forall P \in \mathrm{Op}_{\mathrm{cl}}^{-n}(M)
$$

with the constant $c_{\rho}>0$ for the subset $\Theta_{n}$ of $\mathcal{V}_{\text {sing }}^{+}$such that $\rho\left((1+\Delta)^{-n / 2}\right)>0$. The set $\Theta_{n}$ of singular traces is larger than the set of Dixmier traces $\left(\mathcal{V}_{1}\right)$. Further, for $\rho \in \Theta_{n}$ we have

$$
\rho\left(f(1+\Delta)^{-n / 2}\right)=\frac{\rho\left((1+\Delta)^{-n / 2}\right)}{\operatorname{Vol}(M)} \int_{M} f d x, \quad \forall f \in L^{\infty}(M),
$$

where $d x$ is the volume form on $M$ (Theorem 4.2).

For any spectral triple $(\mathcal{A}, H, D)$ with $\langle D\rangle^{-n} \in \mathcal{M}_{1, \infty}$ define $\Theta(D)$ to be the subset of $\mathcal{V}_{\text {sing }}^{+}$ such that $\rho\left(\langle D\rangle^{-n}\right)>0$. Define the noncommutative integral

$$
\rho\left(a\langle D\rangle^{-n}\right), \quad a \in \mathcal{A}, \quad \rho \in \Theta(D) .
$$

This defines a family of continuous positive traces on $\mathcal{A}$ (in the implied $C^{*}$-norm) (Corollary 4.1).

Restricting the noncommutative integral to the consideration of $\rho \in \mathcal{V}_{1}$ (the set of Dixmier traces or the set of Hardy-Littlewood-Pólya submajorisation ordered continuous functionals on $m_{1, \infty}$, Section 2.1) enables Lidksii type theorems, residue, and heat kernel theorems, see Section 5 and comments.

The functional

$$
\mathrm{f}(a T), \quad a \in \mathcal{A} \subset \mathcal{L}(H)
$$

where $\mathrm{f}$ is a trace on a two-sided ideal of compact operators $\mathcal{J}$ and $0<T \in \mathcal{J}$, is the general format of noncommutative integration introduced by Connes. Such functionals can be characterised by linear functionals $\sigma: \ell^{\infty} \rightarrow \mathbb{C}$ applied to sequences of "expectation values" $\left\{\left\langle h_{m}, a h_{m}\right\rangle\right\}_{m=1}^{\infty}$ for some orthonormal basis $\left\{h_{m}\right\}_{m=1}^{\infty}$ of eigenvectors of $T$. In particular

$$
\mathrm{f}(a T)=\sigma\left(\left\{\left\langle h_{m}, a h_{m}\right\rangle\right\}_{m=1}^{\infty}\right)
$$

for all $a \in \mathcal{L}(H)$.

For flat torii, both commutative and noncommutative, this implies

$$
\mathrm{f}(a g(\Delta))=\mathrm{f}(g(\Delta)) \tau(a)
$$

for any trace $\mathrm{f}$ on a two-sided ideal $\mathcal{J}$, and $g$ a positive bounded Borel function such that $g(\Delta) \in \mathcal{J}$. Here $\Delta$ is the Laplacian (resp. noncommutative Laplacian) on the torus and $\tau$ is the Lebesgue integral on commutative torii (resp. the unique faithful normal finite trace on the type $\mathrm{II}_{1}$ noncommutative torii).

If $\mathcal{N} \subset \mathcal{L}(H)$ is a von Neumann algebra, a positive compact operator $T$ is $(\mathcal{N}, h)$-dominated if, for some orthonormal basis $\left\{h_{m}\right\}_{m=1}^{\infty}$ of eigenvectors of $T$, there exists $h \in H$ such that $\left\|P h_{m}\right\| \leq\|P h\|$ for all projections $P \in \mathcal{N}$. If $\mathcal{J}$ is any two-sided ideal with positive singular trace $\mathrm{f}$, and $0<T \in \mathcal{J}$ is $(\mathcal{N}, h)$-dominated, then $\mathrm{f}(\cdot T)$ is a positive normal linear functional on $\mathcal{N}$. 


\subsection{List of open questions}

Let $(\mathcal{A}, H, D)$ be a spectral triple (as defined in the first paragraph of Section 1) such that $\langle D\rangle^{-n} \in \mathcal{M}_{1, \infty}$ (as defined at (1.5)).

Open Question 7.1 (from Section 1). If $\mathcal{A}^{\prime \prime}$ contains no finite rank operators is $\Phi_{\omega}$ (the functional in (1.1)) normal?

Open Question 7.2 (from Section 1). What are necessary and sufficient relationships between $\langle D\rangle^{-n}$ and the $*$-algebra $\mathcal{A}$ so that $\Phi_{\omega}$ (the functional in (1.1)) is independent of $\omega$ (for various sets of generalised limits)?

Open Question 7.3 (from Section 3). Is $B \subset S_{\infty}\left(\ell^{\infty}\right)$ satisfying the Cesàro limit property ( from $[27, \S 5]$ ) a necessary and sufficient condition for equality of a set $\mathcal{K}_{B}^{+}$of positive measurable operators (e.g. (3.3)) with the Tauberian operators?

Open Question 7.4 (from Section 3). Is $\mathcal{K}_{P D L[1, \infty)}^{+}($defined in (3.3)) equal to the Tauberian operators?

Open Question 7.5 (from Section 3.2). Is there a maximal closed symmetric subideal $\mathcal{U}_{2}$ of $\mathcal{M}_{1, \infty}$ such that $\tilde{\mathcal{T}} \cap \mathcal{U}_{2}=\mathcal{K}_{2} \cap \mathcal{U}_{2}$ ? Is $\mathcal{L}^{1, w} \subseteq \mathcal{U}_{2}$ ?

Open Question 7.6. What is the relation between the sets $\mathcal{V}_{\frac{1}{2}}$ and $\Theta(D)$ (defined above) of singular traces? What are their sets of measurable operators?

\section{A Identifications in (2.37)}

We start with preliminaries. Define

$$
\alpha_{g}(t):=\frac{1}{\log (1+t)} \int_{1}^{t} g^{*}(s) d s, \quad t \geq 1
$$

where

$$
g^{*}(s):=\inf \{t \mid \mu(|g|>t)<s\}, \quad \mu \text { is Lebesgue measure on }[1, \infty) .
$$

Set

$$
\mathrm{m}_{1, \infty}:=\left\{g \in L^{\infty}([1, \infty)) \mid \alpha_{g} \in C_{b}([1, \infty))\right\}
$$

Define: $p$ from $\ell^{\infty}$ to bounded Borel functions on $[0, \infty)$ by

$$
p\left(\left\{a_{k}\right\}_{k=0}^{\infty}\right)(t):=\sum_{k=0}^{\infty} a_{k} \chi_{[k, k+1)}(t), \quad t \geq 0 ;
$$

$p_{c}$ from $\ell^{\infty}$ to continuous bounded functions on $[0, \infty)$ by

$$
p_{c}\left(\left\{a_{k}\right\}_{k=0}^{\infty}\right)(t):=\sum_{k=0}^{\infty}\left(a_{k}+(t-k)\left(a_{k+1}-a_{k}\right)\right) \chi_{[k, k+1)}(t), \quad t \geq 0 ;
$$

$r$ from bounded Borel or continuous functions on $[0, \infty)$ to $\ell^{\infty}$ by

$$
r(f)_{k}:=f(k), \quad k \in\{0,1,2, \ldots\}
$$


$E: L^{\infty}([0, \infty)) \rightarrow L^{\infty}([0, \infty))$ by

$$
E(f)(t):=\int_{t}^{t+1} f(s) d s, \quad t \geq 0
$$

$L^{-1}: L^{\infty}([1, \infty)) \rightarrow L^{\infty}([0, \infty))$ by

$$
L^{-1}(g)(t)=g\left(e^{t}\right), \quad t \geq 0
$$

and finally the maps on $\ell^{\infty}$ for $j \in \mathbb{N}$ by

$$
\begin{aligned}
& T_{j}\left(\left\{a_{k}\right\}_{k=0}^{\infty}\right):=\left\{a_{k+j}\right\}_{k=0}^{\infty}, \\
& C\left(\left\{a_{k}\right\}_{k=0}^{\infty}\right):=\left\{\frac{1}{n+1} \sum_{k=0}^{n} a_{k}\right\}_{n=0}^{\infty},
\end{aligned}
$$

and the maps on $L^{\infty}([0, \infty))$ for $a>0$ by

$$
\begin{aligned}
T_{a}(f)(t) & :=f(t+a), \\
C(f)(t) & :=\frac{1}{t} \int_{0}^{t} f(s) d s .
\end{aligned}
$$

Lemma A.1. Let $\left\{a_{n}\right\}_{n=0}^{\infty} \in \ell^{\infty}$ and $f \in L^{\infty}([0, \infty))$. With the above definitions we have:

1. $\lim _{t \rightarrow \infty}\left(T_{j} p_{(c)}-p_{(c)} T_{j}\right)\left(\left\{a_{n}\right\}\right)(t)=0$;

2. $\lim _{t \rightarrow \infty}\left(C p_{(c)}-p_{(c)} C\right)\left(\left\{a_{n}\right\}\right)(t)=0$;

3. $\left(T_{j} r E-r E T_{j}\right)(f) \in c_{0}$;

4. $(C r E-r E C)(f) \in c_{0}$,

for any $j \in \mathbb{N}$.

Proof. Let $\left\{a_{k}\right\}_{k=0}^{\infty} \in \ell^{\infty}$. Then

$$
\left(T_{j} p-p T_{j}\right)\left(\left\{a_{k}\right\}\right)(t)=\sum_{k=0}^{\infty} a_{k} \chi_{[k, k+1)}(t+j)-\sum_{k=0}^{\infty} a_{k+j} \chi_{[k, k+1)}(t)=0 .
$$

Similarly,

$$
\left(T_{j} p_{c}-p_{c} T_{j}\right)\left(\left\{a_{k}\right\}\right)(t)=0 .
$$

This proves 1 . We also have

$$
\begin{aligned}
(C p-p C)\left(\left\{a_{k}\right\}\right)(t) & =\frac{1}{t} \int_{0}^{t} \sum_{k=0}^{\infty} a_{k} \chi_{[k, k+1)}(s) d s-\sum_{k=0}^{\infty} \frac{1}{k+1} \sum_{i=0}^{k} a_{i} \chi_{[k, k+1)}(t) \\
& =\left(\frac{\lfloor t\rfloor+1}{t}-1\right)\left(C\left(\left\{a_{k}\right\}\right)(\lfloor t\rfloor)+\left(1-\frac{\lfloor t\rfloor}{t}\right) a_{\lfloor t\rfloor} .\right.
\end{aligned}
$$

Hence

$$
\left\|(C p-p C)\left\{a_{k}\right\}\right\|_{\infty} \leq\left(\left|\frac{\lceil t\rceil}{t}-1\right|+\left|\frac{\lfloor t\rfloor}{t}-1\right|\right)\|a\|_{\infty} \rightarrow 0 .
$$


Similarly,

$$
\begin{aligned}
\left(C p_{c}-\right. & \left.p_{c} C\right)\left(\left\{a_{k}\right\}\right)(t)=\frac{1}{t} \int_{0}^{t} \sum_{k=0}^{\infty}\left(a_{k}+(s-k)\left(a_{k+1}-a_{k}\right)\right) \chi_{[k, k+1)}(s) d s \\
& -\sum_{n=0}^{\infty} C\left(\left\{a_{k}\right\}\right)(n)+(t-n)\left(C\left(\left\{a_{k}\right\}\right)(n+1)-C\left(\left\{a_{k}\right\}\right)(n)\right) \chi_{[n, n+1)}(t) \\
= & \frac{1}{t} \sum_{k=0}^{\lfloor t\rfloor} \frac{1}{2}\left(a_{k}+a_{k+1}\right)-\frac{1}{t} \int_{t}^{\lceil t\rceil} a_{\lfloor t\rfloor}+(s-\lfloor t\rfloor)\left(a_{\lfloor t\rfloor+1}-a_{\lfloor t\rfloor}\right) d s \\
& -C\left(\left\{a_{k}\right\}\right)(\lfloor t\rfloor)-(t-\lfloor t\rfloor)\left(C\left(\left\{a_{k}\right\}\right)(\lfloor t\rfloor+1)-C\left(\left\{a_{k}\right\}\right)(\lfloor t\rfloor)\right) .
\end{aligned}
$$

We recall that

$$
C\left(\left\{a_{k}\right\}\right)(n+1)-C\left(\left\{a_{k}\right\}\right)(n)=\left(\frac{n+1}{n+2}-1\right) C\left(\left\{a_{k}\right\}\right)(n)-\frac{a_{n+1}}{n+2}
$$

so that

$$
\lim _{n \rightarrow \infty}\left|C\left(\left\{a_{k}\right\}\right)(n+1)-C\left(\left\{a_{k}\right\}\right)(n)\right|=0 .
$$

Also

$$
C\left(\frac{1}{2}\left(\left\{a_{k+1}+a_{k}\right\}\right)\right)(n)=\frac{1}{2}\left(\frac{a_{n+1}}{n+1}-\frac{a_{0}}{n+1}\right)+C\left(\left\{a_{k}\right\}\right)(n)
$$

so that

$$
\lim _{n \rightarrow \infty}\left|C\left(\frac{1}{2}\left(\left\{a_{k+1}+a_{k}\right\}\right)\right)(n)-C\left(\left\{a_{k}\right\}\right)(n)\right|=0 .
$$

Now

$$
\begin{aligned}
\left\|\left(C p_{c}-p_{c} C\right)\left\{a_{k}\right\}\right\|_{\infty} \leq & \left|\frac{\lceil t\rceil}{t}-1\right|\|a\|_{\infty}+\left|C\left\{\frac{1}{2}\left(a_{k+1}+a_{k}\right)\right\}(\lfloor t\rfloor)-C\left(\left\{a_{k}\right\}\right)(\lfloor t\rfloor)\right| \\
& +\left|C\left(\left\{a_{k}\right\}\right)(\lfloor t\rfloor+1)-C\left(\left\{a_{k}\right\}\right)(\lfloor t\rfloor)\right|+3 \frac{\|a\|_{\infty}}{t} \rightarrow 0 .
\end{aligned}
$$

These results demonstrate 2 .

Set $E^{\prime}=r E: L^{\infty}([0, \infty)) \rightarrow \ell^{\infty}$. Consider

$$
\left(T_{j} E^{\prime}-E^{\prime} T_{j}\right) f(n)=\int_{n}^{n+1} f(t+j) d t-\int_{n+j}^{n+j+1} f(t) d t=0 .
$$

We have

$$
\begin{aligned}
\left|\left(E^{\prime} C-C E^{\prime}\right) f(n)\right| & =\left|\int_{n}^{n+1} \frac{1}{t} \int_{0}^{t} f(s) d s d t-\frac{1}{n+1} \sum_{i=0}^{n} \int_{i}^{i+1} f(s) d s\right| \\
& =\left|\int_{n}^{n+1} \frac{1}{t} \int_{0}^{t} f(s) d s d t-\frac{1}{n+1} \int_{0}^{n+1} f(s) d s\right| \\
& \leq \sup _{t \in[n, n+1)}\left|\frac{1}{t} \int_{0}^{t} f(s) d s-\frac{1}{n+1} \int_{0}^{n+1} f(s) d s\right| \\
& \leq\left(\frac{n+1}{n}-1+\frac{1}{n}\right)\|f\|_{\infty} \rightarrow 0 .
\end{aligned}
$$

These equations demonstrate 3 and 4 . 
Lemma A.2. If $g \in \mathrm{m}_{1, \infty}$, then:

1. $\xi\left(1-p_{(c)} r\right) L^{-1}\left(\alpha_{g}\right)=0$ for all $\xi \in B L_{(c)}[0, \infty)$;

2. $\xi^{\prime} r(1-E) L^{-1}\left(\alpha_{g}\right)=0$ for all $\xi^{\prime} \in B L$.

Proof. The following arguments were first published in a more general form in $[27, \S 2.2]$. We include them for completeness. Let $f=L^{-1}\left(\alpha_{g}\right) \in C_{b}([0, \infty))$ and $c(s)=\log \left(1+e^{s}\right)$. Set

$$
k(s)=\frac{1}{c(s)} \int_{e^{s}}^{e^{s+1}} g^{*}(u) d u, \quad s \geq 0 .
$$

Let $\theta$ be a state of $C_{b}([0, \infty))$ such that $\theta=\theta T_{j}$ for $j \in \mathbb{N}$. For example, $\theta=\left.\xi\right|_{C_{b}([0, \infty))}$ where $\xi \in B L[0, \infty), \theta=\xi$ where $\xi \in B L_{c}[0, \infty)$, or $\xi^{\prime} r$ for $\xi^{\prime} \in B L$. Then

$$
\begin{aligned}
\theta(k) & =\theta\left(\frac{1}{c(s)} \int_{e^{s}}^{e^{s+1}} g^{*}(u) d u\right)=\theta\left(\frac{1}{c(s)} \int_{1}^{e^{s+1}} g^{*}(u) d u\right)-\theta\left(\frac{1}{c(s)} \int_{1}^{e^{s}} g^{*}(u) d u\right) \\
& =\theta\left(\frac{1}{c(s)} \int_{1}^{e^{s+1}} g^{*}(u) d u\right)-\theta\left(\frac{1}{c(s+1)} \int_{1}^{e^{s+1}} g^{*}(u) d u\right) \\
& =\theta\left(\left(1-\frac{c(s+1)}{c(s)}\right) f(s+1)\right)=\lim _{s \rightarrow \infty}\left(1-\frac{c(s+1)}{c(s)}\right) \theta(f)=0 .
\end{aligned}
$$

Now set

$$
K(s)=\sup _{t \in[s, s+1)}|f(t)-f(s)|, \quad s \geq 0 .
$$

Then

$$
\begin{aligned}
K(s) & =\sup _{t \in[s, s+1)}\left|\frac{1}{c(t)} \int_{1}^{e^{t}} g^{*}(u) d u-\frac{1}{c(s)} \int_{1}^{e^{s}} g^{*}(u) d u\right| \\
& \leq \frac{1}{c(s)} \int_{1}^{e^{s+1}} g^{*}(u) d u-\frac{1}{c(s+1)} \int_{1}^{e^{s}} g^{*}(u) d u \\
& \leq \frac{1}{c(s)} \int_{e^{s}}^{e^{s+1}} g^{*}(u) d u+\left(1-\frac{c(s)}{c(s+1)}\right) \frac{1}{c(s)} \int_{1}^{e^{s}} g^{*}(u) d u \\
& \leq k(s)+\left|1-\frac{c(s)}{c(s+1)}\right| f(s) .
\end{aligned}
$$

Hence

$$
\theta(K)=0 .
$$

We have

$$
\begin{aligned}
|\theta((1-p r)(f))| & =|\theta(f(t)-f(\lfloor t\rfloor))|=\mid \theta(f(t-1)-f(\lfloor t\rfloor) \mid \\
& \leq \theta(|f(t-1)-f(\lfloor t\rfloor)|) \leq \theta(K(t-1))=\theta(K)=0 .
\end{aligned}
$$

Also

$$
\begin{aligned}
\left|\theta\left(\left(1-p_{c} r\right)(f)\right)\right| & =|\theta((t-\lfloor t\rfloor)(f(t)-f(\lceil t\rceil))+(1-(t-\lfloor t\rfloor))(f(t)-f(\lfloor t\rfloor)))| \\
& \leq \theta(|f(t)-f(\lceil t\rceil)|)+\theta(|f(t-1)-f(\lfloor t\rfloor)|) \\
& \leq \theta(K(t))+\theta(K(t-1))=2 \theta(K)=0 .
\end{aligned}
$$

This demonstrates 1 . 
Similarly

$$
|(1-E)(f)(s)|=\left|f(s)-\int_{s}^{s+1} f(t) d t\right| \leq \sup _{t \in[s, s+1)}|f(t)-f(s)|=K(s) .
$$

Hence

$$
|\theta((1-E)(f))| \leq \theta(|(1-E)(f)|) \leq \theta(K)=0 .
$$

This demonstrates 2 .

Lemma A.3. For every $\xi \in B L_{(c)}[0, \infty)\left(\right.$ resp. $\left.C B L_{(c)}[0, \infty), B(C)_{(c)}[0, \infty)\right)$ there exists $\xi^{\prime} \in$ $B L($ resp. $C B L, B(C))$ (and for every $\xi^{\prime} \in B L$ (resp. $C B L, B(C)$ ) there exists $\xi \in B L_{(c)}[0, \infty)$ (resp. $\left.\left.C B L_{(c)}[0, \infty), B(C)_{(c)}[0, \infty)\right)\right)$ such that $\left(\xi-\xi^{\prime} r\right) L^{-1}\left(\alpha_{g}\right)(t)=0 \forall g \in \mathrm{m}_{1, \infty}$.

Proof. Let $\xi \in B L_{(c)}[0, \infty)$. Set $\xi^{\prime}=\xi p_{(c)}$. It is easily verified $\xi^{\prime}$ is a state on $\ell^{\infty}$ which is translation invariant by Lemma A.1.1. Moreover,

$$
\left(\xi-\xi^{\prime} r\right) L^{-1}\left(\alpha_{g}\right)=\xi\left(1-p_{(c)} r\right) L^{-1}\left(\alpha_{g}\right)=0
$$

due to Lemma A.2. Now let $\xi^{\prime} \in B L$. Set $\xi=\xi^{\prime} r E$. It is easily verified $\xi$ is a state on $L^{\infty}([0, \infty))$ (resp. $C_{b}([0, \infty))$ ) which is translation invariant for $a \in \mathbb{N}$ by Lemma A.1.3. Let $a=j+k$ where $j \in \mathbb{N}$ and $k \in(0,1)$. Then

$$
\int_{n+k}^{n+1+k} f(s) d s=\int_{n+k}^{n+1} f(s) d s+\int_{n+1}^{n+1+k} f(s) d s
$$

and

$$
\begin{aligned}
\xi^{\prime} r E\left(T_{k} f\right) & =\xi^{\prime}\left(\int_{n+k}^{n+1} f(s) d s\right)+\xi^{\prime}\left(\int_{n+1}^{n+1+k} f(s) d s\right) \\
& =\xi^{\prime}\left(\int_{n+k}^{n+1} f(s) d s\right)+\xi^{\prime}\left(\int_{n}^{n+k} f(s) d s\right)=\xi^{\prime}\left(\int_{n}^{n+1} f(s) d s\right)=\xi^{\prime} r E(f) .
\end{aligned}
$$

Hence $\xi^{\prime} r E$ is translation invariant for all $a>0$. Moreover,

$$
\left(\xi-\xi^{\prime} r\right) L^{-1}\left(\alpha_{g}\right)=\xi^{\prime} r(1-E) L^{-1}\left(\alpha_{g}\right)=0
$$

due to Lemma A.2.

Now let $\xi=\gamma C \in C B L_{(c)}[0, \infty)$ for any singular state $\gamma$ on $L^{\infty}([0, \infty))\left(\right.$ resp. $\left.C_{b}([0, \infty))\right)$. Then

$$
\xi^{\prime}=\xi p_{(c)}=\gamma C p_{(c)}=\gamma p_{(c)} C
$$

by Lemma A.1.2 where $\gamma p_{(c)}$ is a singular state on $\ell^{\infty}$. Hence $\xi^{\prime} \in C B L$. Similarly, if $\xi \in$ $B(C)_{(c)}[0, \infty)$,

$$
\xi^{\prime} C=\xi p_{(c)} C=\xi C p_{(c)}=\xi p_{(c)}=\xi^{\prime}
$$

by Lemma A.1.2 again. Hence $\xi^{\prime} \in B(C)$. Conversely, if $\xi^{\prime}=\gamma^{\prime} C \in C B L$ with $\gamma^{\prime}$ a singular state on $\ell^{\infty}$ then

$$
\xi=\xi^{\prime} r E=\gamma^{\prime} C r E=\gamma^{\prime} r E C
$$

by Lemma A.1.4 where $\gamma^{\prime} r E$ is a singular state on $L^{\infty}([0, \infty))$ (resp. $\left.C_{b}([0, \infty))\right)$. Similarly, if $\xi^{\prime} \in B(C)$,

$$
\xi C=\xi^{\prime} r E C=\xi^{\prime} C r E=\xi^{\prime} r E=\xi
$$

by Lemma A.1.4 again. Hence $\xi \in B(C)_{(c)}[0, \infty)$. 
Corollary A.1. We have the equalities as in (2.39), (2.40) and (2.41).

Proof. We note $g=\sum_{n=1}^{\infty} \mu_{n}(T) \chi_{[n, n+1)} \in \mathrm{m}_{1, \infty}$ for all $T \in \mathcal{M}_{1, \infty}$. The result for (2.39) follows from the diagram

$$
\begin{aligned}
& \mathcal{V}_{D L[1, \infty)} \quad \mathcal{V}_{D L_{c}[1, \infty)} \\
& L \text { ॥ } \quad L \text { ॥ } \\
& \mathcal{W}_{B L[0, \infty)} \stackrel{A .3}{=} \mathcal{W}_{B L} \stackrel{A .3}{=} \quad \mathcal{W}_{B L_{c}[0, \infty)}
\end{aligned}
$$

where $L$ denotes the isomorphism between $\mathcal{W}_{B L[1, \infty)}\left(\right.$ resp. $\left.\mathcal{W}_{B L_{c}[1, \infty)}\right)$ and $\mathcal{V}_{D L[1, \infty)}$ (resp.

$\left.\mathcal{V}_{D L_{c}[1, \infty)}\right)$ induced by the map $L^{-1}(\mathrm{~A} .8)$, and $\stackrel{A .3}{=}$ denotes the statement of Lemma A.3. The result for (2.40) and (2.41) follows from an identical diagram.

\section{Acknowledgements}

The authors thank N. Kalton for many discussions concerning Dixmier traces and symmetric functionals.

\section{References}

[1] Chamseddine A.H., Connes A., The spectral action principle, Comm. Math. Phys. 186 (1997), 731-750, hep-th/9606001.

[2] Connes A., Noncommutative geometry, Academic Press, Inc., San Diego, CA, 1994.

[3] Connes A., Gravity coupled with matter and the foundation of non-commutative geometry, Comm. Math. Phys. 182 (1996), 155-176, hep-th/9603053.

[4] Segal I.E., A non-commutative extension of abstract integration, Ann. of Math. (2) 57 (1953), $401-457$.

[5] Kunze R.A., $L_{p}$ Fourier transforms on locally compact unimodular groups, Trans. Amer. Math. Soc. 89 (1958), 519-540.

[6] Stinespring W.F., Integration theorems for gages and duality for unimodular groups, Trans. Amer. Math. Soc. 90 (1959), 15-56.

[7] Nelson E., Notes on non-commutative integration, J. Funct. Anal. 15 (1974), 103-116.

[8] Fack T., Kosaki H., Generalised s-numbers of $\tau$-measurable operators, Pacific J. Math. 123 (1986), $269-300$.

[9] Pisier G., Xu Q., Non-commutative $L^{p}$-spaces, in Handbook of the geometry of Banach spaces, Vol. 2, North-Holland, Amsterdam, 2003, 1459-1517.

[10] Pederson G.K., $C^{*}$-algebras and their automorphism groups, London Mathematical Society Monographs, Vol. 14, Academic Press, London - New York, 1979.

[11] Simon B., Trace ideals and their applications, 2nd ed., Mathematical Surveys and Monographs, Vol. 120, American Mathematical Society, Providence, RI, 2005.

[12] von Neumann J., Some matrix inequalities and metrization of metric-space, Rev. Tomsk. Univ. 1 (1937), 286-300.

[13] Bratteli O., Robinson D.W., Operator algebras and quantum statistical mechanics. 1. $C^{*}$ - and $W^{*}$-algebras, symmetry groups, decomposition of states, 2nd ed., Texts and Monographs in Physics, Springer-Verlag, New York, 1987.

[14] Cipriani F., Guido D., Scarlatti S., A remark on trace properties of K-cycles, J. Operator. Theory 35 (1996), 179-189, funct-an/9506003.

[15] Carey A.L., Rennie A., Sedaev A., Sukochev F., The Dixmier trace and asymptotics of zeta functions, J. Funct. Anal. 249 (2007), 253-283, math.OA/0611629.

[16] Benameuar M.-T., Fack T., Type II non-commutative geometry. I. Dixmier trace in von Neumann algebras, Adv. Math. 199 (2006), 29-87.

[17] Connes A., The action functional in noncommutative geometry, Comm. Math. Phys. 117 (1988), 673-683.

[18] Dunford N., Schwartz J.T., Linear operators. Part I. General theory, John Wiley \& Sons, Inc., New York, 1988. 
[19] Carey A.L., Sukochev F.A., Dixmier traces and some applications in noncommutative geometry, Russian Math. Surveys 61 (2006), 1039-1099, math.OA/0608375.

[20] Guido D., Isola T., Dimensions and singular traces for spectral triples, with applications to fractals, J. Funct. Anal. 203 (2003), 362-400, math.OA/0202108.

[21] Pietsch A., About the Banach envelope of $l_{1, \infty}$, Rev. Mat. Complut. 22 (2009), 209-226.

[22] Dixmier J., Existence de traces non normales, C. R. Acad. Sci. Paris Sér. A-B 262 (1966), A1107-A1108.

[23] Lorentz G.G., A contribution to the theory of divergent sequences, Acta. Math. 80 (1948), 167-190.

[24] Sucheston L., Banach limits, Amer. Math. Monthly 74 (1967), 308-311.

[25] Carey A., Phillips J., Sukochev F., Spectral flow and Dixmier traces, Adv. Math. 173 (2003), 68-113. math.OA/0205076.

[26] Krein S.G., Petunin Yu.I., Semenov E.M., Interpolation of linear operators, Translations of Mathematical Monographs, Vol. 54, American Mathematical Society, Providence, R.I., 1982.

[27] Lord S., Sedaev A., Sukochev F., Dixmier traces as singular symmetric functionals and applications to measurable operators, J. Funct. Anal. 244 (2005), 72-106, math.FA/0501131.

[28] Dodds P.G., de Pagter B., Semenov E.M., Sukochev F.A., Symmetric functional and singular traces, Positivity 2 (1998), 47-75.

[29] Dodds P.G., de Pagter B., Sedaev A.A., Semenov E.M., Sukochev F.A., Singular symmetric functionals, Zap. Nauchn. Sem. S.-Peterburg. Otdel. Mat. Inst. Steklov. (POMI) 290 (2002), 42-71 (English transl.: J. Math. Sci. 2 (2004), 4867-4885).

[30] Dodds P.G., de Pagter B., Sedaev A.A., Semenov E.M., Sukochev F.A., Singular symmetric functionals and Banach limits with additional invariance properties, Izv. Ross. Akad. Nauk Ser. Mat. 67 (2003), no. 6, 111-136 (English transl.: Izv. Math. 67 (2003), 1187-1212).

[31] Guido D., Isola T., Singular traces on semifinite von Neumann algebras, J. Funct. Anal. 134 (1995), 451-485.

[32] Sedaev A.A., Generalized limits and related asymptotic formulas, Math. Notes 86 (2009), 577-590.

[33] Sedaev A.A., Sukochev F.A., Zanin D.V., Lidskii-type formulae for Dixmier traces, Integral Equations Operator Theory, to appear, arXiv:1003.1813.

[34] Semenov E.M., Sukochev F.A., Invariant Banach limits and applications, J. Funct. Anal. 259 (2010), 15171541.

[35] Azamov N.A., Sukochev F.A., A Lidskii type formula for Dximier traces, C. R. Math. Acad. Sci. Paris 340 (2005), 107-112.

[36] Prinzis R., Traces résiduelles et asymptotique du spectre d'opérateurs pseduo-différentiels, Ph.D. Thesis, Lyon, 1995.

[37] Calkin J.W., Two-sided ideals and congruences in the ring of bounded operators in Hilbert space, Ann. of Math. (2) 42 (1941), 839-873.

[38] Kalton N.J., Sukochev F.A., Symmetric norms and spaces of operators, J. Reine Angew. Math. 621 (2008), 81-121.

[39] Hardy G.H., Littlewood J.E., Pólya G., Inequalities, Cambridge University Press, Cambridge, 1934.

[40] Kalton N., Sukochev F., Rearrangement-invariant functionals with applications to traces on symmetrically normed ideals, Canad. Math. Bull. 51 (2008), 67-80.

[41] Albeverio S., Guido D., Ponosov A., Scarlatti S., Singular traces and compact operators, J. Funct. Anal. 137 (1996), 281-302, funct-an/9308001.

[42] Gracia-Bondía J.M., Várilly J.C., Figueroa H., Elements of noncommutative geometry, Birkhäuser Advanced Texts: Basler Lehrbücher, Birkhäuser Boston, Inc., Boston, MA, 2001.

[43] Wodzicki M., Local invariants of spectral asymmetry, Invent. Math. 75 (1984), 143-178.

[44] Shubin M.A., Pseudodifferential operators and spectral theory, 2nd ed., Springer-Verlag, Berlin, 2001.

[45] Landi G., An introduction to noncommutative spaces and their geometries, Lecture Notes in Physics, New Series m: Monographs, Vol. 51, Springer-Verlag, Berlin, 1997.

[46] Hörmander L., The analysis of linear partial differential operators. III. Pseudo-differential operators, Grundlehren der Mathematischen Wissenschaften, Vol. 274, Springer-Verlag, Berlin, 1994.

[47] Connes A., Moscovici H., The local index formula in noncommutative geometry, Geom. Funct. Anal. 5 (1995), 174-243. 
[48] Carey A.L., Philips J., Rennie A., Sukochev F.A., The Hochschild class of the Chern character for semifinite spectral triples, J. Funct. Anal. 213 (2004), 111-153, math.OA/0312073.

[49] Carey A.L., Philips J., Rennie A., Sukochev F.A., The local index formula in semifinite von Neumann algebras. I. Spectral flow, Adv. Math. 202 (2006), 451-516, math.OA/0411019.

[50] Carey A.L., Philips J., Rennie A., Sukochev F.A., The local index formula in semifinite von Neumann algebras. II. The even case, Adv. Math. 202 (2006), 517-554, math.OA/0411021.

[51] Carey A., Potapov D., Sukochev F., Spectral flow is the integral of one forms on the Banach manifold of self adjoint Fredholm operators, Adv. Math. 222 (2009), 1809-1849, arXiv:0807.2129.

[52] Higson N., The residue index theorem of Connes and Moscovici, in Surveys in Noncommutative Geometry, Clay Math. Proc., Vol. 6, Amer. Math. Soc., Providence, RI, 2006, 71-126.

[53] Ponge R., Noncommutative geometry and lower dimensional volumes in Riemannian geometry, Lett. Math. Phys. 83 (2008), 19-32, arXiv:0707.4201.

[54] Guido D., Isola T., Noncommutative Riemann integration and Novikov-Shubin invariants for open manifolds, J. Funct. Anal. 176 (2000), 115-152, math.OA/9802015.

[55] Englis M., Guo K., Zhang G., Toeplitz and Hankel operators and Dixmier traces on the unit ball of $\mathbb{C}^{n}$, Proc. Amer. Math. Soc. 137 (2009), 3669-3678, arXiv:0707.2025.

[56] Nicola F., Rodino L., Dixmier traceability for general pseudo-differential operators, in $C^{*}$-Algebras and Elliptic Theory II, Trends Math., Birkhäuser, Basel, 2008, 227-237.

[57] Nest R., Schrohe E., Dixmier's trace for boundary value problems, Manuscripta Math 96 (1998), $203-218$.

[58] Pearson J., Bellissard J., Noncommutative Riemannian geometry and diffusion on ultrametric Cantor sets, J. Noncommut. Geom. 3 (2009), 447-480, arXiv:0802.1336.

[59] Lapidus M.L., Toward a noncommutative fractal geometry, Laplacians and volume measures on fractals, in Harmonic Analysis and Nonlinear Differential Equations (Riverside, CA, 1995), Contemp. Math., Vol. 208, Amer. Math. Soc., Providence, RI, 1997, 211-252.

[60] Rudin W., Invariant means on $L^{\infty}$, in Collection of Articles Honoring the Completion by Antoni Zygmund of 50 Years of ScientifIc Activity. III, Studia Math. 44 (1972), 219-227.

[61] Lord S., Potapov D., Sukochev F., Measures from Dixmier traces and zeta functions, J. Funct. Anal. 259 (2010), 1915-1949, arXiv:0905.1172.

[62] Lindenstrauss J., Tzafriri L., Classical Banach spaces. II. Function spaces, Ergebnisse der Mathematik und ihrer Grenzgebiete, Vol. 97, Springer-Verlag, Berlin - New York, 1979.

[63] Wodzicki M., Noncommutative residue. I. Fundamentals, in K-Theory, Arithmetic and Geometry (Moscow, 1984-1986), Lecture Notes in Math., Vol. 1289, Springer, Berlin, 1987, 320-399.

[64] Seeley R.T., Complex powers of an elliptic operator, Singular Integrals (Proc. Sympos. Pure Math., Chicago, Ill., 1966), Amer. Math. Soc., Providence, R.I., 1967, 288-307.

[65] Minakshisundaram S., Pleijel A., Some properties of the eigenfunctions of the Laplace-operator on Riemannian manifolds, Canad. J. Math. 1 (1949), 242-256.

[66] Higson N., Meromorphic continuation of zeta functions associated to elliptic operators, Operator Algebras, Quantization, and Noncommutative Geometry, Contemp. Math., Vol. 365, Amer. Math. Soc., Providence, RI, 2004, 129-142.

[67] Gilkey P.B., Invariance theory, the heat equation, and the Atiyah-Singer index theorem, 2nd ed., Studies in Advanced Mathematics, CRC Press, Boca Raton, FL, 1995.

[68] Berline N., Getzler E., Vergne M., Heat kernels and Dirac operators, Grundlehren der Mathematischen Wissenschaften, Vol. 298, Springer-Verlag, Berlin, 1992.

[69] Connes A., Geometry from the spectral point of view, Lett. Math. Phys. 34 (1995), 203-238.

[70] Lord S., Sukochev F.A., Noncommutative residues and a characterisation of the noncommutative integral, Proc. Amer. Math. Soc., to appear, arXiv:0905.0187.

[71] Kalton N.J., Spectral characterization of sums of commutators. I, J. Reine Angew. Math. 504 (1998), 115125, math.FA/9709209.

[72] Dykema K.J., Figiel T., Weiss G., Wodzicki M., Commutator structure of operator ideals, Adv. Math. 185 (2004), 1-79.

[73] Donnelly H., Quantum unique ergodicity, Proc. Amer. Math. Soc. 131 (2003), 2945-2951.

[74] Sarnak P., Arithmetic quantum chaos, in The Schur Lectures (Tel Aviv, 1992), Israel Math. Conf. Proc., Vol. 8, Bar-Ilan Univ., Ramat Gan, 1995, 183-236.

[75] Rieffel M.A., $C^{*}$-algebras associated with irrational rotations, Pacific J. Math. 93 (1981), 415-429. 